\title{
Effect of $D$ on the evolution of radiation damage in $W$ during high temperature annealing
}

\author{
M. Pečovnik ${ }^{* a}$, S. Markelja ${ }^{\mathrm{a}}$ M. Kelemen ${ }^{\mathrm{a}}$, T. Schwarz-Selinger ${ }^{\mathrm{b}}$ \\ a Jožef Stefan Institute, Jamova cesta 39, 1000 Ljubljana, Slovenia \\ ${ }^{b}$ Max-Planck-Institut für Plasmaphysik, Boltzmannstrasse 2, D-85748 Garching, Germany
}

\begin{abstract}
The effect of deuterium (D) on the annealing of radiation damage was studied. Tungsten (W) samples were sequentially irradiated with $20 \mathrm{MeV} \mathrm{W}$ ions at room temperature and loaded with a low-temperature D plasma at $370 \mathrm{~K}$ to decorate the created defects. After this, half of each sample was $\mathrm{W}$ irradiated at room temperature again to create additional radiation damage. To study the evolution of the created defects with $\mathrm{D}$ being present, samples were annealed by heating them to a desired temperature and held for two hours. The surviving displacement damage was decorated by re-exposing the samples to the same D plasma as before. At various steps of the experiment the D depth profiles were measured using Nuclear Reaction Analysis and after the last re-exposure a Thermal Desorption Spectroscopy analysis was done to determine the D desorption spectra.

D release is dominated by two desorption peaks centered at $520 \mathrm{~K}$ and $780 \mathrm{~K}$ for a $3 \mathrm{~K} / \mathrm{min}$ temperature ramp. For temperature holds below $500 \mathrm{~K}$ no measurable change in the intensity of the desorption peaks was measured. For temperature holds above $500 \mathrm{~K}$, the low temperature D desorption peak started to reduce in intensity. The high temperature D desorption peak showed signs of evolution only for temperature holds above $600 \mathrm{~K}$. This behaviour was independent of the number of $\mathrm{W}$ irradiations used.

A macroscopic rate equation model was used to recreate the experimental results. Using the fill-level dependant D-defect interaction picture we were able to determine that three distinct defect types with several fill-levels each are sufficient to describe the results. The concentrations of defect 1 (vacancies) and defect 2 (small vacancy clusters) reduced by $50 \%$ and $35 \%$, respectively, after an $800 \mathrm{~K}$ temperature hold. No reduction was found for defect 3 (large vacancy clusters).
\end{abstract}

Keywords: tungsten, deuterium retention, displacement damage, macroscopic rate-equations

\section{PACS:}

*Corresponding author: matic.pecovnik@ijs.si

\section{Introduction}


In future fusion reactors one of the main material candidates for the plasma-facing surfaces is W, as it shows favourable properties such as high melting temperature, high thermal conductivity and low intrinsic retention of hydrogen isotopes (HI). In a future tokamak environment its crystal lattice will be severely damaged by the $14 \mathrm{MeV}$ neutrons produced in the D-T fusion reaction creating defects in the crystal lattice of tungsten $[1,2]$. These defects will increase the retained amount of HIs in the material by orders of magnitude because of the strong binding energy of HI to the defects. This means that if we wish to understand HI transport and retention in W we must understand the evolution of defects after they have been created. To study the evolution of radiation-induced defects, heavy ion irradiation with $\mathrm{MeV}$ energy is usually used [3] as it only introduces displacement damage without causing material activation, making the material much easier to handle in a subsequent laboratory analysis.

It is clear that in order to fully understand the influence of displacement damage on HI retention, one must understand the evolution of defects at elevated temperatures. In the recent past, several studies were conducted to address this in detail [4-6]. However, in all these cases the annealing of displacement damage in hydrogen-free tungsten was studied. Little is known how the presence of HIs could affect the evolution of defects. In recent experiments [7-9], W samples were irradiated with $\mathrm{MeV} \mathrm{W}$ ions with and without the presence of $\mathrm{D}$. The created displacement damage was decorated with D plasma [7]/atoms [8]/ions [9] which acted as a tracer for the underlying defects that were created during irradiation. The experimental results showed that substantially more displacement damage was created if the $\mathrm{W}$ irradiation occurred with $\mathrm{D}$ already present in the material. This so-called stabilization effect was further elaborated on by simulations using macroscopic rate equations [10], [11]. The simulation efforts were built upon DFT studies which previously showed that if $\mathrm{D}$ is trapped in vacancy-type defects it can reduce the likelihood of Frenkel-pair annihilation in the case of single-vacancies in $b c c-\mathrm{W}$ [12] and increases the binding energies of vacancy-clusters in $b c c-F e$ [13]. This stabilization effect during displacement damage creation suggests that the presence of $\mathrm{D}$ in $\mathrm{W}$ could affect the evolution of defects also during annealing. In this work, annealing of displacement damage refers to heating the sample to a desired temperature and holding it there for a specific amount of time in order to study the annihilation and evolution of existing defects in the material.

In this work we will describe an experiment where the effect of annealing of defects at elevated temperatures was studied where the tungsten samples contained deuterium during 
annealing. Hence defect evolution takes place with D being present. After the experiment was performed the experimental results were simulated by a simplified one-dimensional rate-equation code which allowed us to determine the concentrations of defects that survive the annealing procedure.

\section{Experiment}

In the experiment polycrystalline $99.997 \mathrm{wt}$ \% hot-rolled tungsten samples manufactured by Plansee were used. They have been taken from the same batch as the W samples used in our

previous experiments [7-9]. They were $12 \times 15 \mathrm{~mm}^{2}$ in size and $0.8 \mathrm{~mm}$ thick. Grains were elongated in the rolling direction being parallel to the surface. The surfaces were mechanically polished down to $5 \mu \mathrm{m}$ grit and subsequently electro-polished in $1.5 \% \mathrm{NaOH}$ solution to a mirrorlike finish. In order to reduce the density of natural defects present in the as received material, the samples were heated for $2 \mathrm{~min}$ in vacuum at $2000 \mathrm{~K}$ for re-crystallization after polishing. This procedure enlarges the grain size to $10-50 \mu \mathrm{m}$.

\subsection{Experimental Procedure}

The $\mathrm{W}$ samples were irradiated by $20 \mathrm{MeV} \mathrm{W}^{6+}$ ions at room temperature $(295 \mathrm{~K})$ using the $3 \mathrm{MV}$ Tandetron ion accelerator at the Max-Planck-Institut for Plasma Physics (IPP), Garching. The chosen energy of the $\mathrm{W}$ ion irradiation creates displacement damage that extends to about $2.3 \mu \mathrm{m}$. The primary damage depth distribution has been calculated using SRIM [14] and is shown in Fig. 1a and Fig. 1b. The samples were irradiated using a W fluence of $7.87 \times 10^{17} \mathrm{~W} / \mathrm{m}^{2}$, which converts to a calculated damage dose at the peak maximum of 0.23 dракР (Kinchin-Pease calculation, $90 \mathrm{eV}$ displacement damage energy, evaluating the "vacancy.txt" output). Such a fluence was chosen as we expect that it should be sufficient to achieve displacement damage saturation in terms of D retention throughout the entire damage layer up to the calculated maximum $\mathrm{W}$ ion range [15]. Homogeneous implantation over the entire surface area of the sample was ensured by scanning the W beam over the whole sample area [7].

After W irradiation, the samples were loaded with a well-characterized low-temperature D plasma $(\mathrm{PlaQ})$ at an exposure temperature of $370 \mathrm{~K}$ for 72 hours. The $\mathrm{D}$ ion flux used was $5.6 \times 10^{19} \mathrm{D} / \mathrm{m}^{2}$ s yielding a $\mathrm{D}$ fluence of $1.5 \times 10^{25} \mathrm{D} / \mathrm{m}^{2}$. The tungsten samples were mounted on a floating target holder which results in the energy of the impacting D ions equal to $15 \mathrm{eV}$. Because 
approximately $94 \%$ of the flux consists of $\mathrm{D}_{3}{ }^{+}$ions the energy of the ions hitting the sample surface is considered as $<5 \mathrm{eV} / \mathrm{D}$ [16]. Such an energy is below the displacement energy threshold for tungsten, which is why we can assume that no additional displacement damage is created during the D exposure. From [17] subthreshold damage caused by the low-energy D ion exposure itself is also expected to be negligible for the chosen exposure parameters.

The influence of further $\mathrm{W}$ implantation was studied. From recent experiments and simulations [7-11], it was inferred that D presence during W ion irradiation causes stabilization of displacement damage induced defects. This is why half of each $\mathrm{W}$ sample was irradiated again after the first D plasma exposure. This was achieved by covering each sample by a molybdenum mask, that ensured that half of the sample was covered. The $\mathrm{W}$ irradiation procedure was identical as described above.

Afterwards the samples were sent to Jožef Stefan Institute (JSI). There, the samples were ramped to a desired temperature $(400,500,600,800$ and $1000 \mathrm{~K})$ and held there for 2 hours in a vacuum chamber where the base pressure was below $10^{-8} \mathrm{mbar}$ and did not increase above $2 \times 10^{-7}$ mbar during annealing even at the highest annealing temperature. The heating ramp used to heat the samples to the desired temperature was $30 \mathrm{~K} / \mathrm{min}$. The temperature of the sample and heater are monitored in real-time using a pair of thermocouples attached to the sample and heater directly. From the measurement of the sample temperature we have determined that the actual heating rate of the sample was $24 \mathrm{~K} / \mathrm{min}$. During the ramp and hold procedure the signal of desorbing D was monitored using a residual gas analyser (RGA) (Pfeiffer, Prisma Plus quadrupole mass spectrometer, 1-100 amu/q). The amount of desorbing $\mathrm{D}$ was derived by summing the contribution from $\mathrm{HD}$ and $\mathrm{D}_{2}$ molecules ( $3 \mathrm{amu} / \mathrm{q}$ and $4 \mathrm{amu} / \mathrm{q}$, respectively), assuming equal sensitivities. Desorption of D is also possible in the form of deuterated water - HDO (19 amu/q) and $\mathrm{D}_{2} \mathrm{O}(20 \mathrm{amu} / \mathrm{q})$ - but their contribution could not be distinguished from the background contribution and are therefore ignored. For more details on the apparatus used in this part of the experiment, please see [19]. The D desorption signal was measured during the entire process. After the samples were cooled down in vacuum the $\mathrm{D}$ depth profiles were measured to determine the amount of retained D that remained captured in the sample after the elevated temperature hold.

After annealing the D depth profile was measured on both halves by Nuclear Reaction Analysis (NRA). These samples were then re-exposed in PlaQ at the same exposure conditions as before to populate the defects that survived the annealing process. After this re-exposure, NRA analysis was done on both halves of each sample again to determine the amount of $\mathrm{D}$ in the samples. This 
procedure allows to determine the relative loss of defects due to the temperature hold because the amount of surviving defects is directly correlated to the measured amount of D in the samples. Afterwards, the samples were cut in half to separate the single-damaged side which was W irradiated once before annealing from the double-damaged side which was W irradiated twice. Cutting was performed along the short side of the sample with a water-cooled saw equipped with a diamond blade to prevent D loss. After the entire $\mathrm{W}$ irradiation and D loading sequence Thermal Desorption Spectroscopy (TDS) was carried out on both halves of each sample. This specific experimental procedure was chosen, as it closely resembles the one used by Markina et al. [4], which will allow us to compare our results, where D was present during annealing, to those presented in Ref. [4] where no D was present during annealing. The only important difference between both experiments is that in [4] a 1-hour long annealing hold was chosen. Based on the results presented therein, we have decided to use a 2-hour long annealing hold to ensure that steadystate in terms of defect evolution would occur at all annealing temperatures.

In addition to the samples that were $\mathrm{W}$ irradiated, $\mathrm{D}$ exposed and annealed in the manner described above, we also show experimental results from Ref. [7]. The samples used in that study were prepared, W irradiated and D exposed in exactly the same manner as the samples of the

present study, with the exception that the samples were not annealed. For this reason we have used some of the data available in Ref. [7] to complement our own. The specific data will be highlighted when used.

After analysis of the D depth profile and D desorption data for the sample held at $1000 \mathrm{~K}$ and $\mathrm{D}$ re-exposed we have discovered that the $\mathrm{D}$ did not penetrate to the maximum damage depth after the second D exposure performed after annealing because of an unknown reason. The loading of the other samples which were exposed in PlaQ at the same time was found to be without issues. To achieve complete damage layer saturation, the D re-exposure was redone after the TDS but the damage layer was still not saturated. Because of this its experimental results could not be used. The only usable experimental result of the $1000 \mathrm{~K}$ annealed sample is its D desorption data during the ramp and hold.

\subsection{Experimental techniques}

Two main experimental techniques were used to characterize the D retention at several steps in the experimental procedure. 
The NRA technique allowed the determination of the depth distribution of $\mathrm{D}$ at the selected times before annealing, after annealing and after the D re-exposure in PlaQ. The nuclear reaction $\mathrm{D}\left({ }^{3} \mathrm{He}, \alpha\right) \mathrm{p}$ was used to determine the $\mathrm{D}$ depth profile. The ${ }^{3} \mathrm{He}$ analysing beam was $2 \mathrm{~mm}$ in diameter. Six analysing beam energies were used ranging from 0.7 to $4.2 \mathrm{MeV}$. The proton detector used for the NRA measurement was mounted at a 135-degree reaction angle. For more details on the NRA analysing technique, see [9]. The output of the NRA measurement are the raw proton energy spectra, which were input into NRADC [20] that outputs the most probable D depth profile.

The D desorption spectra were measured using TDS. This measurement allows us to infer the amount of surviving defect densities after the annealing process and $\mathrm{D}$ re-exposure in PlaQ through the use of rate-equation models. The TDS measurement was performed in the TESS set-up at IPP using a linear oven heating ramp of $3 \mathrm{~K} / \mathrm{min}$ up to $1010 \mathrm{~K}$ and a $>30 \mathrm{~min}$ hold at the highest temperature. To determine the amount of $\mathrm{D}$ desorbed during the measurement masses $3 \mathrm{amu} / \mathrm{q}$ and $4 \mathrm{amu} / \mathrm{q}$, corresponding to $\mathrm{HD}$ and $\mathrm{D}_{2}$ molecules respectively, were summed up. The calibration factor for $\mathrm{D}_{2}$ was determined using a leak bottle from Laco Technologies with a flow of $1.22 \times 10^{14} \mathrm{D}_{2} / \mathrm{s}$ and a stated accuracy of $4.6 \%$. The calibration factor for HD was experimentally determined by flowing either $\mathrm{D}_{2}$ or HD gas through an orifice of known size from a calibrated volume into the mass spectroscopy vessel. The amount of D desorbed in the form of other species like $\mathrm{D}_{2} \mathrm{O}$ and $\mathrm{HD}$ cannot be distinguished from the background contribution and is therefore ignored. The sample temperature during the TDS measurement was monitored directly by using a shielded thermocouple that was in direct contact with the sample. For more details on the TDS measurement please see Ref. [7]. The absolute D amounts obtained by integrating the D desorption spectrum for both the single- and double-damaged half of each sample were weighted by the ratio of the masses for each half. This was done in order to compensate for the small differences in the non-perfect cuts in the middle of each sample.

\section{Experimental Results}

\subsection{WD-AD experimental series}

The $\mathrm{D}$ depth profiles and $\mathrm{D}$ desorption spectra for the annealing series of the single-damaged samples will be presented first. The experimental series will be denoted as WD-AD, the abbreviation stemming from $(\mathrm{W})$ irradiation $+(\mathrm{D})$ loading $+(\mathrm{A})$ nnealing $+(\mathrm{D})$ loading. The $\mathrm{D}$ 
depth profiles are shown in Figs. 1a-e and the D desorption spectra are shown in Fig. 1f. In Figs. 1a-e also the primary damage distribution calculated by SRIM is shown in grey. We see that the peak damage dose of $0.23 \mathrm{dpa}$ occurs at a depth of around $1.3 \mu \mathrm{m}$ and the damaging profile extends to $2.3 \mu \mathrm{m}$.

The D depth profile measured on the single damaged side before annealing is shown in Fig. 1a. This measurement was made on the same sample that was later annealed at $600 \mathrm{~K}$. Its D depth distribution is relatively homogeneous throughout the entire calculated damage depth, although a clear decrease in D concentration can be found between 0-0.5 $\mu \mathrm{m}$ compared to the $\mathrm{D}$ concentration measured between $0.5-2.0 \mu \mathrm{m}$. It appears that the $\mathrm{W}$ fluence used to create the displacement damage was not enough to achieve saturation in the entire damaged layer. Still, the relative homogeneity of the D distribution means that all of the defects created down to a depth of $2.3 \mu \mathrm{m}$ were successfully decorated with the D fluence used in our experiment. The same shape of the D depth distribution can be seen in all $\mathrm{D}$ depth profiles.

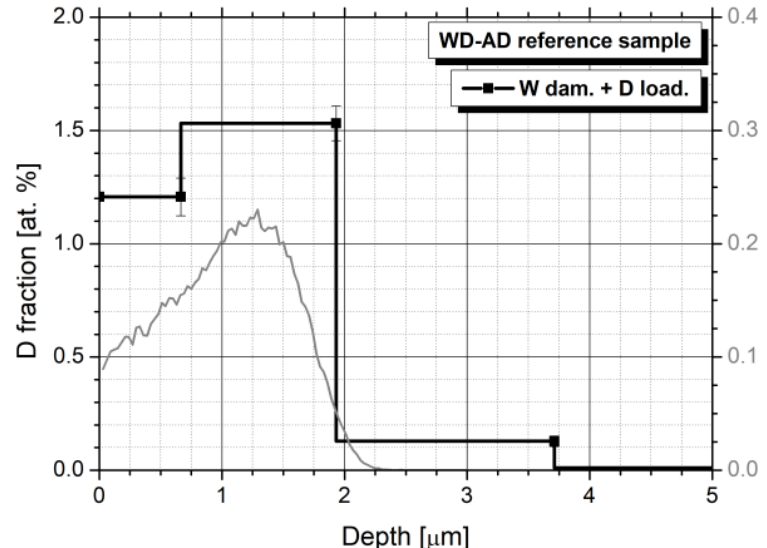

(a)

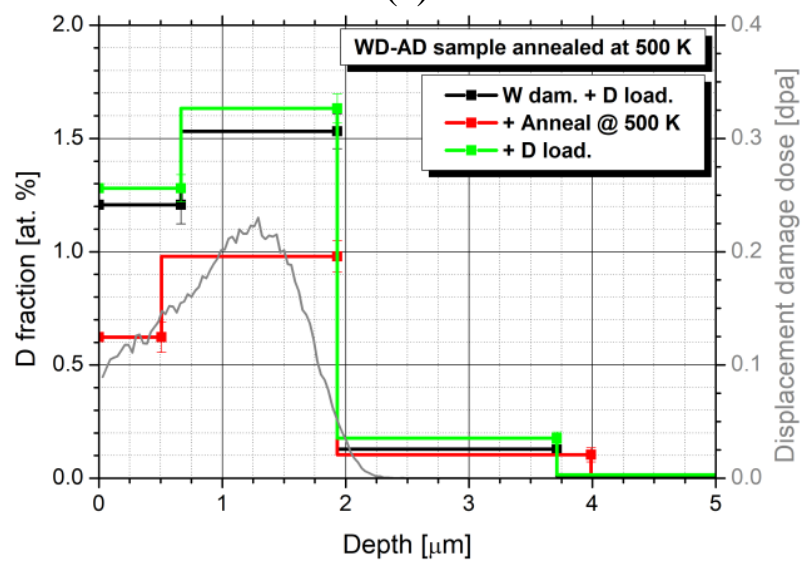

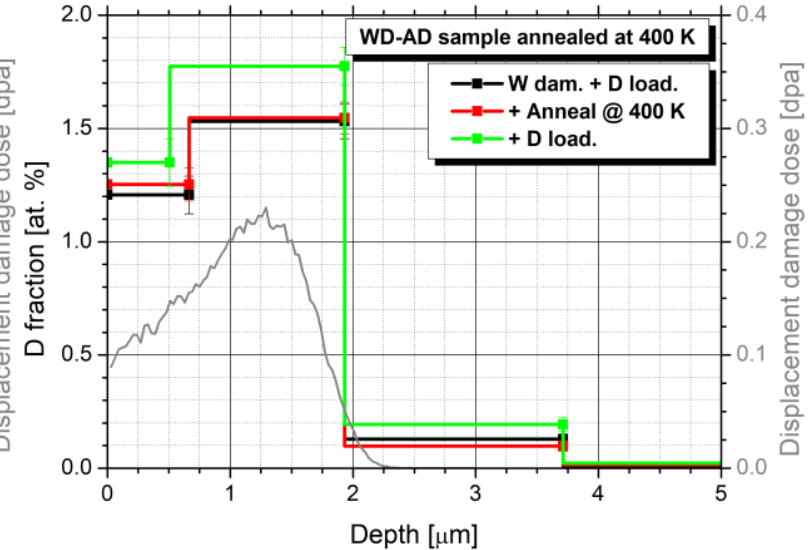

(b)

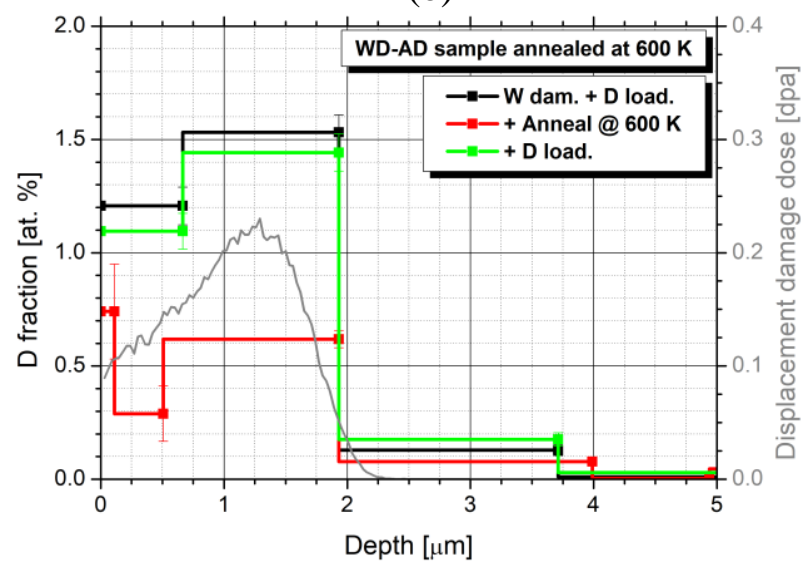


(c)

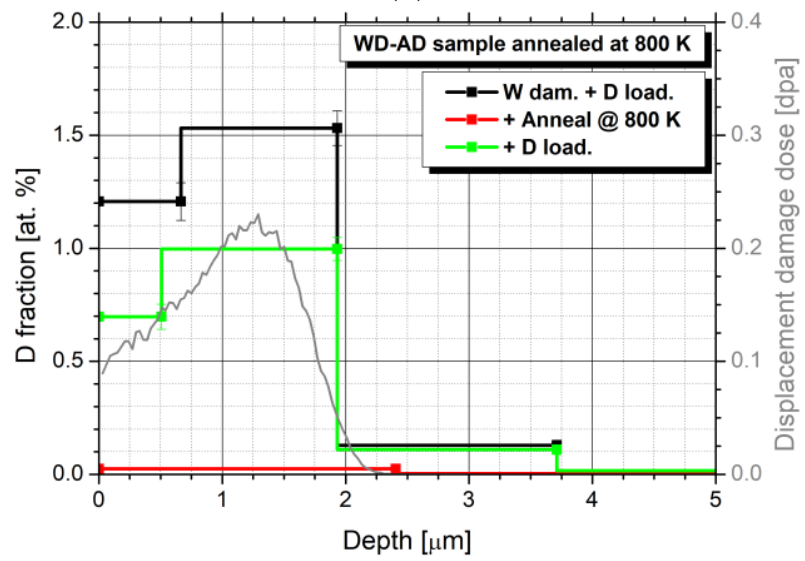

(e) (d)

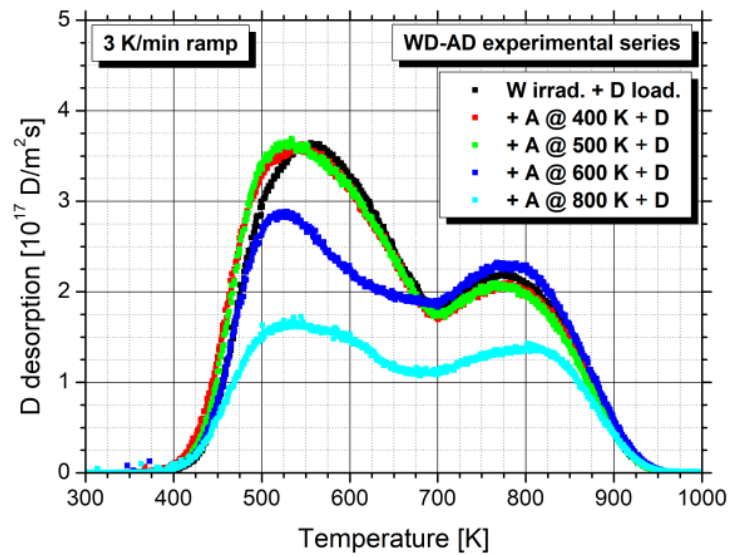

(f)

Figure 1: D depth profiles and D desorption spectra (3 K/min temperature ramp), for the WD-AD experimental series. Samples were exposed to D plasma at $370 \mathrm{~K}$ to a fluence of $1.5 \times 10^{25} \mathrm{D} / \mathrm{m}^{2}$ before and after annealing. D depth profiles are shown for: (a) no annealing, (b) $400 \mathrm{~K},(\mathrm{c}) 500 \mathrm{~K},(\mathrm{~d}) 600 \mathrm{~K}$ and (e) $800 \mathrm{~K}$ annealing. The black $\mathrm{D}$ depth profile shown in all figures represents the reference sample which was W irradiated and D loaded only once measured on the sample which was later annealed at $600 \mathrm{~K}$. The red $D$ depth profile was measured after the annealing procedure. The green $D$ depth profile was measured after the D plasma re-exposure and is the actual D concentration from which we can infer the densities of the surviving defects. In the D depth profile figures the SRIM calculated primary damage profile is also shown in grey. The D desorption spectra (f) are shown for all the samples and were measured after the re-exposure to D plasma. The reference $D$ desorption spectrum drawn in black is taken from Ref. [7] as no TDS measurement was performed on an non-annealed sample. The error bars shown in the D depth profile only take into account the uncertainty of the $D$ depth profile deconvolution and the uncertainty that comes from the limited proton counts that were measured using NRA.

The D depth profiles measured after annealing are plotted in red for each respective annealing temperature. By comparing the maximum D concentration of the D depth profile measured after annealing (red) to the D depth profile of the reference sample (black) we can infer the amount of retained $\mathrm{D}$ that was desorbed during annealing. When annealing at $400 \mathrm{~K}$ no reduction in maximum D concentration is observed. At 500, 600, and $800 \mathrm{~K}$ a $30 \%, 63 \%$ and $100 \%$ reduction in maximum D concentration is observed, respectively.

The most important D depth profiles shown in Figs. 1a-e are the D depth profiles after the $\mathrm{D}$ re-exposure (plotted in green). They are important because the $\mathrm{D}$ concentration measured after the $\mathrm{D}$ re-exposure is directly proportional to the density of defects that has survived the 2 hour annealing process. This means that any reduction in $\mathrm{D}$ concentration compared to the black reference D depth profile can be attributed solely to defect evolution. From the D depth profiles of the samples annealed at 400 and $500 \mathrm{~K}$ no reduction in D concentration can be seen. Furthermore, a slight increase is observed at both temperatures. The increase of maximum D concentration in the case of annealing at $500 \mathrm{~K}$ is around $6 \%$, which is within the experimental uncertainty. Meanwhile a $15 \%$ increase in maximum D concentration measured when annealing at $400 \mathrm{~K}$ could 
be considered significant. At $600 \mathrm{~K}$ a reduction in maximum D concentration of about $10 \%$ is observable. At $800 \mathrm{~K}$ the measured reduction in maximum D concentration is approximately $30 \%$.

The experimental D desorption spectra are shown in Fig. 1f for all annealing temperatures. Because the D desorption spectra were measured after the D re-exposure, we can again state that any reduction of $\mathrm{D}$ amount inferred from the $\mathrm{D}$ desorption spectra at various annealing temperatures can be considered as a clear sign of defect evolution. As the sample used for the measurement of the D depth profile before annealing (Fig. 1a) was later annealed at $600 \mathrm{~K}$ no D desorption spectrum is available for a non-annealed sample in our experiment. Therefore we have used the D desorption spectrum from Ref. [7]. This reference data is plotted with black dots. We can see that the reference $\mathrm{D}$ desorption spectrum consists of two very broad peaks centered around 520 and $780 \mathrm{~K}$. No D desorption is observed at temperatures higher than $950 \mathrm{~K}$. When annealing at $400 \mathrm{~K}$ (red) and $500 \mathrm{~K}$ (green) the D desorption spectra are almost completely the same as the reference D desorption spectrum. At $600 \mathrm{~K}$ (blue) a considerable change in the shape of the D desorption spectrum can be seen. The low temperature D desorption peak now contains much less D compared to the reference sample, exhibiting a $32 \%$ reduction in peak intensity, while the high temperature D desorption peak stays relatively unaltered. When annealing at $800 \mathrm{~K}$ (cyan) a large reduction of trapped $\mathrm{D}$ in both $\mathrm{D}$ desorption peaks becomes apparent. Both the low and high temperature D desorption peak reduced by approximately $50 \%$ of the reference value.

\subsection{WDW-AD experimental series}

Now we turn our attention to the sample halves that were $\mathrm{W}$-irradiated for a second time after the first D plasma loading and before the annealing procedure. We shall denote this experimental series as WDW-AD, which stands for (W) irradiation + (D) loading + (W) irradiation + (A)nnealing + (D) loading. There are two main conceptual differences between both series. Mainly, due to the D stabilization effect during the second $\mathrm{W}$ irradiation the amount of initial (before annealing) displacement damage in the WDW-AD series is roughly twice the amount of displacement damage in the WD-AD series [7]. The other important difference is that in the WD-AD series all of the defects created by the first $\mathrm{W}$ irradiation were filled with $\mathrm{D}$ before annealing. In the WDW-AD series around half of the displacement damage was filled with $\mathrm{D}$, while the other half was almost empty of D, because no second D loading was performed before annealing. 
In Fig. 2a the D depth profiles of the sample which was $\mathrm{W}$ irradiated, D plasma exposed and $\mathrm{W}$ irradiated again is shown in grey $(\mathrm{W}+\mathrm{D}+\mathrm{W})$. This $\mathrm{D}$ depth profile was measured on the sample later annealed at $600 \mathrm{~K}$. We can see that the $\mathrm{D}$ depth profile is similar to the $\mathrm{D}$ depth profile obtained in the other half of the sample (WD side). Therefore, as was already observed in [7], the second $\mathrm{W}$ irradiation does not affect the $\mathrm{D}$ depth profile. As this sample was not re-exposed to D plasma after the second irradiation, the actual saturation concentration of $\mathrm{D}$ in the sample before annealing is unknown. However, we can use data from Ref. [7] which is shown in Fig. 2a (drawn in black) and labelled 'Double-damage reference'. We have to stress here that the D depth profile shown in [7] suffers from an unphysical feature at $0.5 \mu \mathrm{m}$. The depth profile shown here is the result of a more accurate re-evaluation of the raw data with a slightly different energy calibration to avoid this feature.

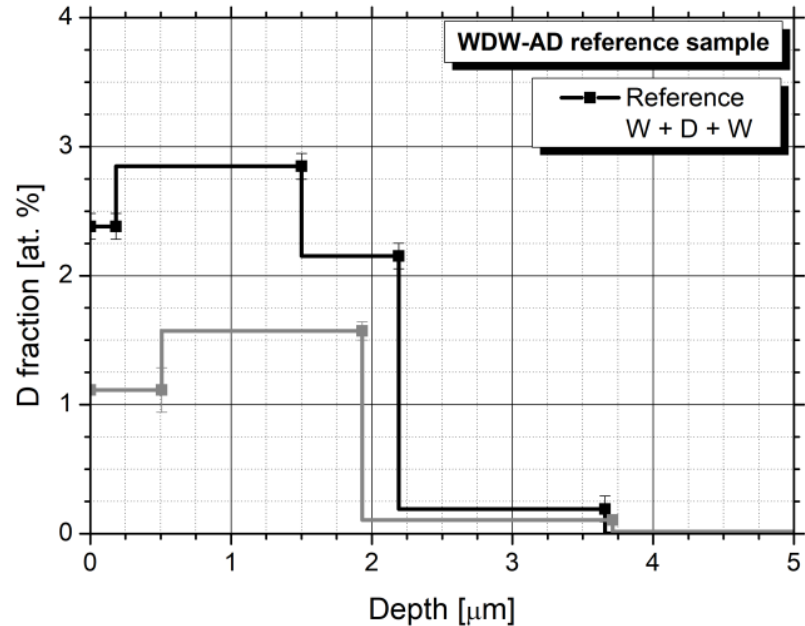

(a)

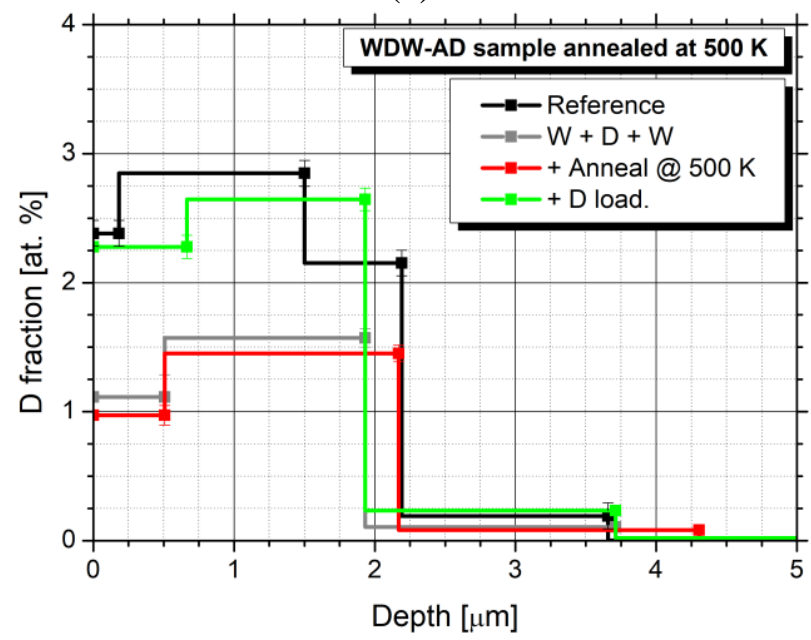

(c)

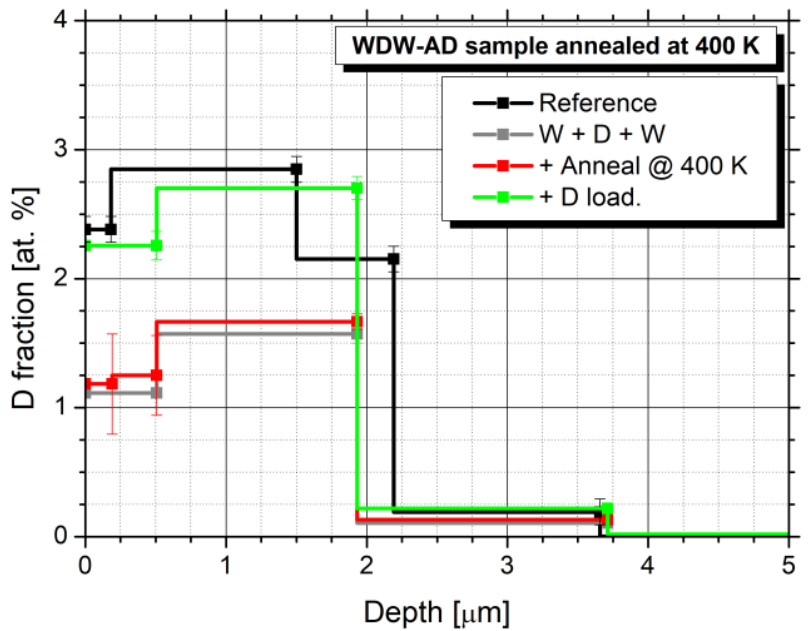

(b)

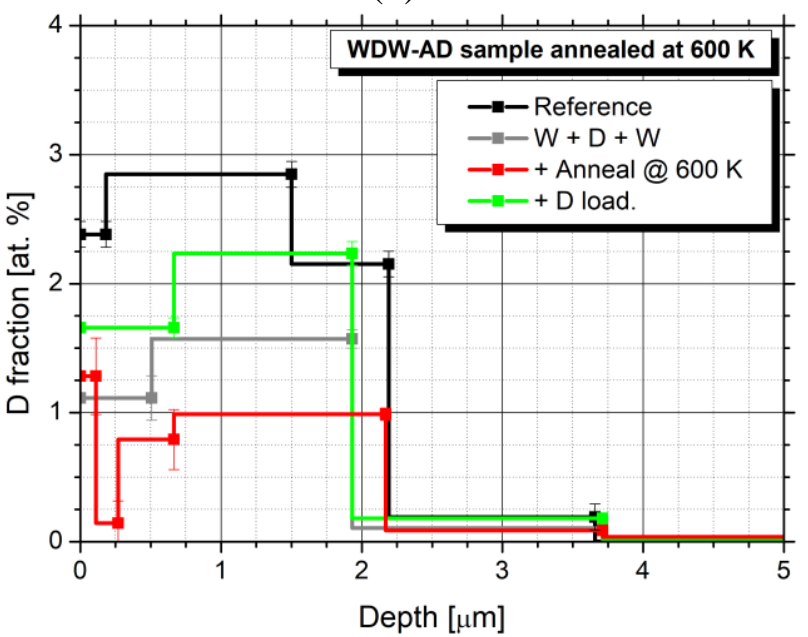

(d) 


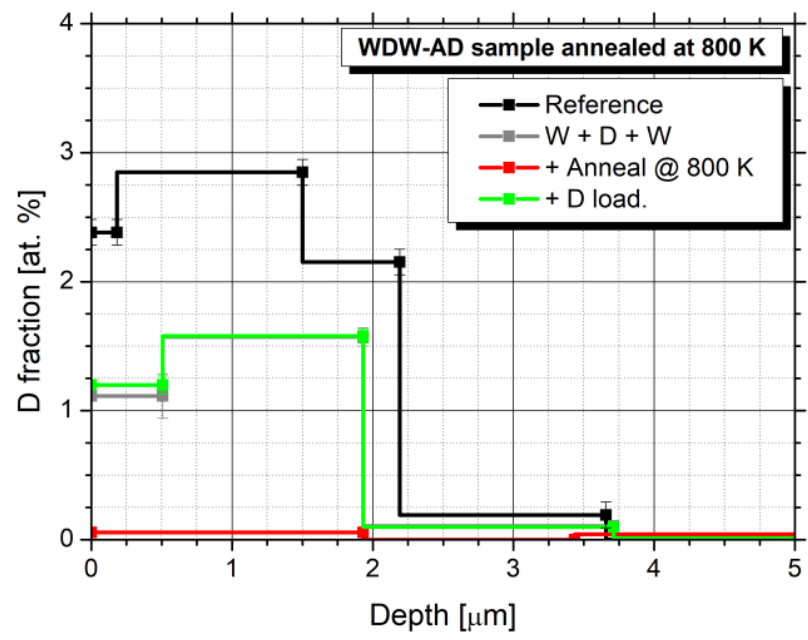

(e)

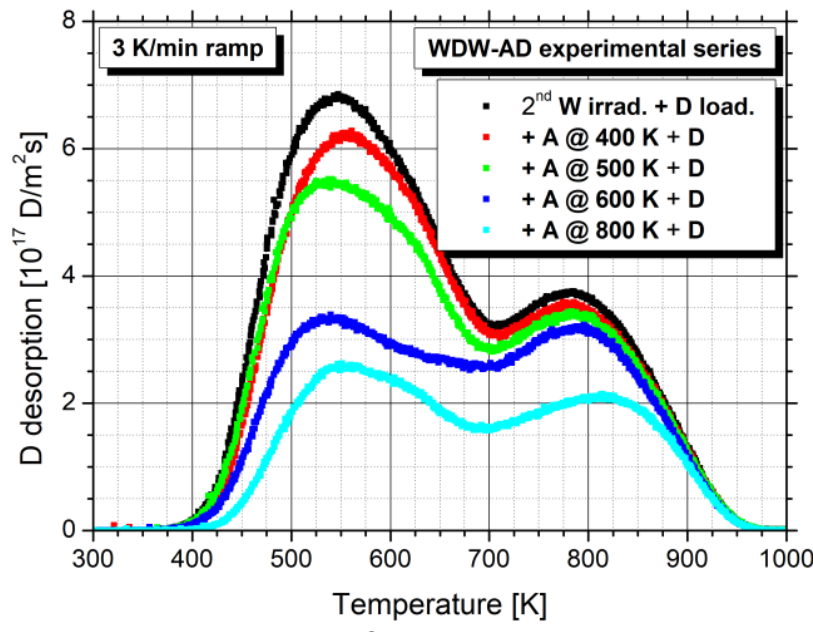

(f)

Figure 2: $D$ depth profiles and D desorption spectra (3 K/min temperature ramp), for the WDW-AD experimental series. Samples were exposed to D plasma at $370 \mathrm{~K}$ to a fluence $1.5 \times 10^{25} \mathrm{D} / \mathrm{m}^{2}$ before the second irradiation and after annealing. D depth profiles are shown for all the annealing temperatures: (a) no annealing, (b) $400 \mathrm{~K}$, (c) $500 \mathrm{~K},(\mathrm{~d}) 600 \mathrm{~K}$ and (e) $800 \mathrm{~K}$. The black D depth profile shown in all figures represents the reference sample which was $W$-irradiated twice and $D$ loaded after each irradiation and was not annealed. This is taken from Ref. [7]. The grey D depth profile was measured after the second irradiation before annealing was performed at $600 \mathrm{~K}$. The red D depth profile were measured after the annealing procedure. The green D depth profile were measured after the D plasma re-exposure and is the actual D concentration from which we can infer the densities of the surviving defects. The $D$ desorption spectra (f) are shown for all the samples. They were measured after the re-exposure to D plasma. The error bars shown in the D depth profile only take into account the uncertainty of the D depth profile deconvolution and the uncertainty that comes from the limited proton counts that were measured using NRA.

Now we can turn to analysing the other D depth profiles which belong to samples which were annealed at various temperatures, shown in Figs. 2b-e. As in the WD-AD experimental series results, D depth profiles plotted with red were measured immediately after the annealing procedure, while the green D depth profiles were measured after the D plasma re-exposure.

First, we analyse the D depth profiles measured after the annealing procedure (red). As expected, no reduction in maximum D concentration is observed when annealing at $400 \mathrm{~K}$ (Fig. 2b). This is the same as in the WD-AD series. At $500 \mathrm{~K}$ (Fig. 2c) a negligible reduction in maximum D concentration is observed in the WDW-AD series, while in the WD-AD series a reduction of approximately $30 \%$ is observed. This difference is attributed to the fact that in the WDW-AD series around half of the displacement damage that was created was empty [7]. As a consequence, there were more empty sites available which meant that weakly bound D that was de-trapped during annealing could be re-captured in them. At $600 \mathrm{~K}$ (Fig. 2d) a reduction in maximum D concentration equal to $33 \%$ was measured compared to $63 \%$ in the respective WDAD series sample. Again, a smaller reduction is observed because more empty sites are available for the D to be re-captured in. When annealing at $800 \mathrm{~K}$ (Fig. 2e) D retention reduced to a level not distinguishable from an undamaged sample hence the reduction is $100 \%$. 
The $\mathrm{D}$ depth profiles measured after the D plasma re-exposure are drawn in green. At 400 and $500 \mathrm{~K}$ a $5 \%$ and $9 \%$ reduction in $\mathrm{D}$ concentration is observed compared to the reference double-damaged sample drawn in black. At 600 and $800 \mathrm{~K}$, the measured reduction in D concentration is $22 \%$ and $45 \%$, respectively. The temperature trend in displacement damage annealing inferred from the reduction in D concentration for both WD-AD and WDW-AD series is therefore quite similar. Almost no reduction is observed at temperatures below $500 \mathrm{~K}$, while a significant reduction is observed for temperatures higher than $500 \mathrm{~K}$.

The experimental D desorption spectra are shown in Fig. 2f. To infer displacement damage evolution from the $\mathrm{D}$ desorption spectra themselves, they must be compared to a $\mathrm{D}$ desorption spectrum of a sample that was $\mathrm{W}$-irradiated twice and $\mathrm{D}$ loaded after each $\mathrm{W}$ irradiation. As no such sample is available in our study, we have used the D desorption spectrum available in Ref. [7], which is plotted in black. As already seen in the D desorption spectra of the WD-AD series, the D desorption spectra consist of a low temperature D desorption peak at around $520 \mathrm{~K}$ and a high temperature desorption peak at around $780 \mathrm{~K}$. All of the retained D is desorbed at temperatures of around $950 \mathrm{~K}$. Comparing the behaviour of the intensity of the D desorption peaks to the behaviour of $\mathrm{D}$ concentration in the $\mathrm{D}$ depth profiles measured after the $\mathrm{D}$ plasma re-exposure, we see that a slightly more pronounced annealing behaviour in the D desorption spectra. Even at the lowest annealing temperature of $400 \mathrm{~K}$ (red) an $8 \%$ reduction in the intensity of the low temperature $\mathrm{D}$ desorption peak and a $7 \%$ reduction in the intensity of the high temperature D desorption peak are observed. When annealing at $500 \mathrm{~K}$ (green), a reduction of $20 \%$ and $11 \%$ in the intensity of the low and high temperature D desorption peaks are observed, respectively. When annealing at $600 \mathrm{~K}$ (blue), almost $50 \%$ of the reference intensity of the low temperature D desorption peak is lost, and $10 \%$ of the intensity of the high temperature D desorption peak. When annealing at $800 \mathrm{~K}$ (cyan), a $62 \%$ and $47 \%$ reduction in $\mathrm{D}$ desorption intensity in the low temperature peak and high temperature D desorption peaks are observed, respectively. Comparing these reductions in intensity observed in the D desorption spectra for the WD-AD and WDW-AD experiment, we can see that the relative loss of $\mathrm{D}$ is slightly larger in the WDW-AD experiment but not by a large margin. Otherwise the general behaviour is similar, as the low temperature D desorption peak starts to exhibit significant annealing at holding temperatures around $500 \mathrm{~K}$, and the high temperature D desorption peak shows significant annealing only at higher holding temperatures i.e. in the $600-800 \mathrm{~K}$ range. 


\subsection{D desorption during Ramp and Hold}

During the annealing of the samples the D desorption signal was monitored. As the D depth profile of all samples prior to annealing is assumed to be the same, the shape and amount of desorbed D up to the specific samples annealing temperature should be the same. That is why the $\mathrm{D}$ desorption signals are normalized in such a way, that the D desorption signals measured for all annealing temperatures match at the low temperature $(530 \mathrm{~K}) \mathrm{D}$ desorption shoulder. The measurements of $\mathrm{D}$ desorption during the ramps can be seen in Fig. 3a and during the holds in Fig. 3b. The sample annealed at $400 \mathrm{~K}$ is not shown as very little D desorption was observed both in the ramp and hold part of the annealing procedure. While analysing the following experimental results we must keep in mind that at this stage half of each sample was already irradiated for a second time but the samples were not cut in half yet, meaning the signal as seen in Fig. 3a and $3 \mathrm{~b}$ is a sum of D desorption from both parts of the sample.

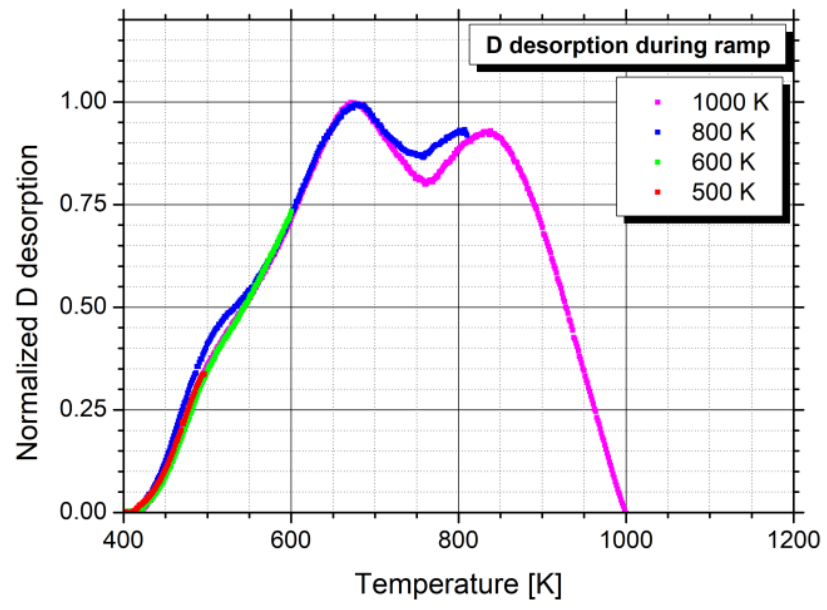

(a)

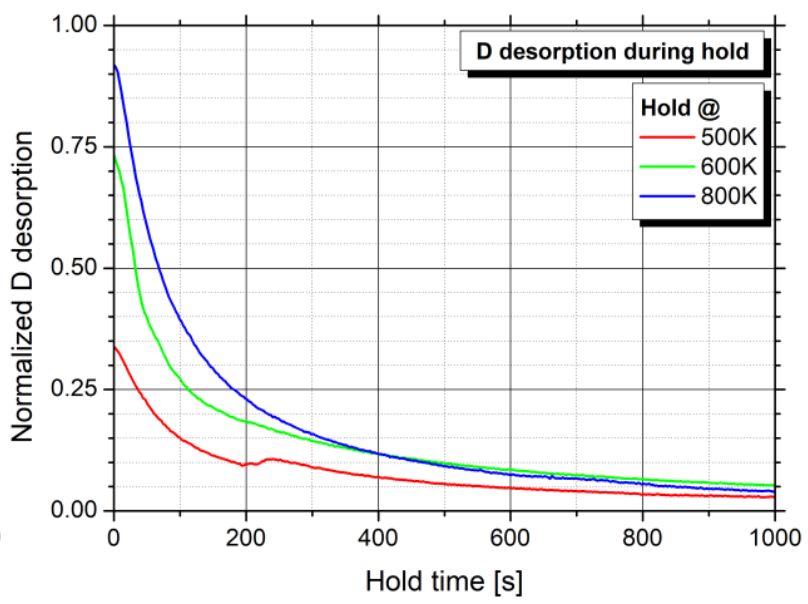

(b)

Figure 3: Normalized D desorption during the ramp and hold of the sample annealing process for a $24 \mathrm{~K} /$ min temperature ramp. The ramp part is shown in (a) and the hold in (b).

The D desorption signals from samples ramped to different temperatures are very similar in shape and behaviour, up to the specific annealing temperature. The $\mathrm{D}$ desorption during the ramp occurs in two distinct peaks at 630 and $830 \mathrm{~K}$ as seen in Fig. 3a. This means that the peaks are shifted to slightly higher temperatures compared to the complete TDS shown in Fig. $1 \mathrm{f}$ and Fig. $2 \mathrm{f}$ where the peaks were centered at 520 and $780 \mathrm{~K}$. This can be explained by two facts. First the faster temperature ramp here shifts the peak temperatures to higher values. The heating rate of the sample was measured to be $24 \mathrm{~K} / \mathrm{min}$, while the heating rate of the TDS shown in Fig. If and $2 \mathrm{f}$ was 3 
$\mathrm{K} / \mathrm{min}$. Second, as was already seen and explained in Fig 2a-e, the second $\mathrm{W}$ irradiation produces new defects. As D is released from the defects with low de-trapping energy (D release in the low temperature desorption peak) they are efficiently re-trapped in the new empty defects. This means they are mainly released in the high temperature D desorption peak, as was already shown in [7]. As the D desorption seen in Fig. 3a is a weighted sum of both the once- and twice-irradiated sample side, the re-capturing of $\mathrm{D}$ results in a lower $\mathrm{D}$ desorption in the low-temperature desorption peak and a higher $\mathrm{D}$ desorption in the high-temperature desorption peak.

In Fig. $3 b$ the D release for the hold part of the procedure are shown, except for holding at $1000 \mathrm{~K}$ as all $\mathrm{D}$ was already lost during the ramp. Other samples show an exponential-like D desorption behaviour with a characteristic time scale in the 200-300 s range. This means that a majority of D that is lost during the hold is lost in the first 10 minutes of the hold. Meanwhile, macroscopic defect evolution has been shown to have a time-scale on the order of tens to hundreds of minutes for steady state to occur. As an example, the annealing behaviour of neutron-induced defects has been determined using resistivity measurements by Keys and Moteff [21]. The authors have shown that the typical time-scale of neutron-induced displacement damage annealing is on the order of hundreds of minutes. In the experiment by Markina et al. [4] where the samples were irradiated with $20 \mathrm{MeV} \mathrm{W}$ ions, the typical annealing time-scale was closer to 30-60 minutes. In any case, we can somewhat safely conclude that the rate of $\mathrm{D}$ desorption during the annealing hold is much faster than the rate of displacement damage annealing. This means that the high concentration of $\mathrm{D}$ that was present in the sample at the beginning of the annealing procedure is not expected to have a significant impact on the measured defect evolution. Furthermore, as a steady-state concentration of $\mathrm{D}$ has been achieved much quicker than a macroscopic defect evolution steady state is expected to occur, we can infer that most of the evolution of the defects occurred at steady-state D occupancies specific to each defect, which can be correlated with the annealing behaviour of each defect.

\subsection{NRA and TDS total D amounts}

In this section we compare the retention of $\mathrm{D}$ in the samples after they have been annealed and reexposed to the D plasma. We compare the retention of $\mathrm{D}$ as measured by both the NRA and TDS techniques. The total $\mathrm{D}$ amounts are plotted as a function of annealing temperature in Fig. 4a. The data points of the non-annealed samples are plotted at $370 \mathrm{~K}$ as they have been exposed to the low- 
temperature D plasma for several tens of hours at this temperature. The total amount of D measured with the TDS technique is systematically about 5-10\% lower than the total amount measured by the NRA technique, except in the case of the non-annealed sample from the WD-AD series. This exception can come from the fact that the NRA data point was measured in this study while the TDS data point was taken from Ref. [7]. In general, the mismatch between TDS and NRA is still in line with the derived experimental uncertainties. When considering uncertainties one should take special note to differentiate between systematic and statistical errors as systematic errors lead to an offset between the NRA and TDS data which we have observed in our data.

The TDS measurement systematic error comes from two main sources. The first stems from the absolute signal calibration with the D leak bottle with a stated uncertainty of $4.6 \%$ (as per manufacturer). The second systematic error stems from the fact we are unable to distinguish D desorption in the form of $\mathrm{HDO}$ and $\mathrm{D}_{2} \mathrm{O}$ from the background contribution. As we are unable to quantitatively determine the systemic error of $\mathrm{HDO}$ and $\mathrm{D}_{2} \mathrm{O}$ contribution we are forced to ignore it. This means the total TDS measurement systematic error is approximately $5 \%$. The systematic error of the NRA measurement stems from the error of the NRA cross section determination (5\% [22]) and the accuracy in determining the accumulated dose equal to $5 \%$ for our setup. Combined, the systematic uncertainty of the NRA measurement is $7.1 \%$. Together the systematic errors of both measuring techniques could account for an offset between the TDS and NRA D retention data equal to $12 \%$. The offset found in our data, which is between 5-10\% falls well within this range.

While the statistical errors do not contribute to an offset between the TDS and NRA data, we must still consider them carefully when analysing our results, as they scatter the data points around their real value. In the case of the TDS measurement, the statistical error comes mainly from the drift and fluctuations of the quadrupole signal and from cutting each sample in half before the measurement to separate the single- and double-damaged side. The error because of the quadrupole signal fluctuations was measured by dosing a constant flux of $\mathrm{D}_{2}$ while monitoring the $4 \mathrm{amu} / \mathrm{q}$ QMS signal. It was determined to be approximately 1-2\%. The statistical error stemming from the cutting of the samples comes mainly from the error of determination of the surface area for each sample half after cutting. A surface area must be assumed if we wish to compare a TDS and NRA measurement as the output of the NRA measurement is an areal density of D $\left[\mathrm{D} / \mathrm{m}^{2}\right]$ while the output of the TDS measurement is the total D amount that was desorbed [D]. Although this surface area is known in principle $\left(7 \times 10 \mathrm{~mm}^{2}\right)$, the complicated experimental procedure with 
multiple $\mathrm{W}$ irradiations, using sample masks for $\mathrm{W}$ irradiation and sample cutting before the TDS measurement introduces an error in determining the exact sample surface area which in turn introduces an uncertainty in the TDS derived D retention of at least a few percent. By combining both of these statistical errors of the TDS measurement we can expect a scatter in our data points of at least 2-3\%. Also the NRA measurement has its own statistical error. These stem from the limited amount of counts we measure at each measuring ${ }^{3} \mathrm{He}$ beam energy and by the uncertainty of the D depth profile fit by NRADC. Both of these together yield a statistical error associated with the NRA measurement of a few percent.

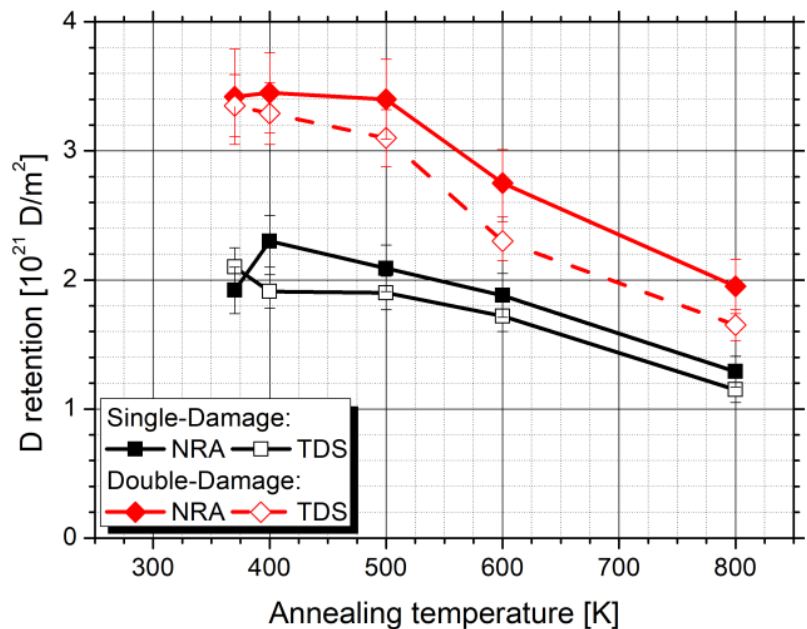

(a)

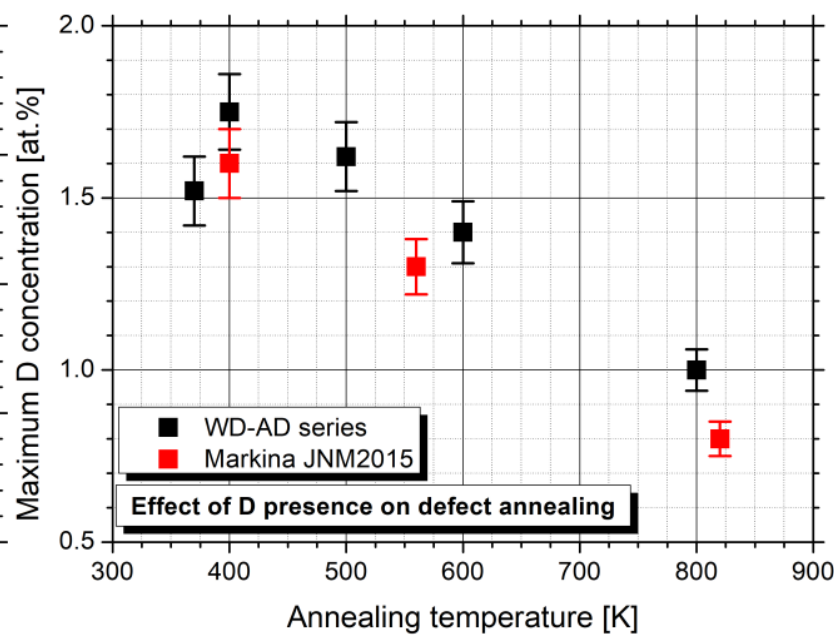

(b)

Figure 4: Comparisons of D retention from integrating the D depth profiles down to $7 \mu$ m (full symbols) or by integrating the D desorption spectra over time (empty symbols) and assuming a $7 \times 10 \mathrm{~mm}^{2}$ area. The WD-AD series is shown in black and the WDW$A D$ is shown in red. (b) Maximum $D$ concentrations measured in our WD-AD (black filled squares) experiments where $D$ was present during annealing are compared to the maximum D concentrations measured by Markina et al. in Ref. [4] (red) where no D was present during annealing. The error bars shown in the D depth profile only take into account the uncertainty of the D depth profile deconvolution and the uncertainty that comes from the limited proton counts that were measured using NRA.

Both D total amounts derived from the NRA and TDS techniques exhibit similar behaviour as function of annealing temperature as can be seen in Fig. 4a. Namely, the total D amount derived with both techniques starts to fall significantly when annealing temperatures reach at least $500 \mathrm{~K}$. At annealing temperatures lower than $500 \mathrm{~K}$ the D total amounts are comparable to the nonannealed reference sample for both measurement techniques. By combining the experimental results for both the WD-AD and WDW-AD experimental series we can conclude that defect annealing starts at annealing temperatures between 500-600 K. First defects that de-trap D in the low D desorption peak start to anneal and are followed in the 600-800 K range by the defects that de-trap D in the high temperature D desorption peak. 
To highlight any possible effect of D presence on defect annealing we compare our WDAD experimental series to the experiment performed by Markina et. al [4]. Their experiment is the closest comparable experiment found in the literature as they have used the same room temperature $20 \mathrm{MeV} \mathrm{W}$ ion irradiation to create displacement damage, and a 1 hour annealing procedure, and the same low-temperature plasma $\mathrm{D}$ exposure with floating target to decorate the surviving defects. However, in their case a $400 \mathrm{~K}$ sample temperature was used for decorating the defects with D. The $30 \mathrm{~K}$ higher exposure temperature might lead to a small reduction in D concentration but we expect it to be within the experimental uncertainties. We must stress that the long D plasma exposures at $370 \mathrm{~K}$ in our experiment and at $400 \mathrm{~K}$ by Markina et. al can be considered as annealing which is why the non-annealed samples must be interpreted accordingly. The comparison between both experiments is drawn in Fig. 4b. As can be seen, the maximum D concentrations when annealing with D present (black squares) are systematically slightly higher compared to the maximum D concentrations when annealing without D (red squares). At annealing temperatures below $800 \mathrm{~K}$ the difference is within the experimental uncertainties. When comparing the $\mathrm{D}$ maximum concentrations when annealing is performed at $800 \mathrm{~K}$ in our experiment and at $820 \mathrm{~K}$ in the experiment by Markina et al. the difference is slightly larger, the maximum D concentrations being $(1 \pm 0.06)$ at. $\%$ and $(0.8 \pm 0.05)$ at. $\%$, respectively. While this difference can be somewhat explained by the higher D exposure temperature and slightly higher temperature of annealing found in the experiment performed by Markina et al., a possibility remains that the presence of D also has an effect. To establish any possible effect of D presence on annealing also the WD-AD and WDW-AD datasets can be compared due to the difference in how much of the displacement damage retained $\mathrm{D}$ prior to annealing. In the case of $\mathrm{WD}-\mathrm{AD}$ all of the created damage was completely saturated with D, while in the case of WDW-AD around half of the created damage was saturated and the other half contained very little D. As can be seen in Fig. 4a only marginal differences can be found in the temperature behaviour of retained $\mathrm{D}$. This in combination with conclusions drawn by comparing the WD-AD dataset to the experiment performed by Markina et al. [4] indicates that the presence of D only has a small effect on displacement damage annealing if present at all. However, the annealing hold of two hours is long compared to the time-scales of defect evolution, therefore we cannot exclude that the D could affect annealing if much shorter holds would have been used. 


\section{Simulation}

In order to understand the annealing behaviour of the defects involved in D retention in our tungsten samples, we will simulate the experimental results.

For this, we use the MHIMS-R code [23], which is based on the fill-level dependent picture of the D-defect interaction [24]. We choose to use the fill-level dependant D-defect interaction picture as we have observed in the previous section, that the low temperature $\mathrm{D}$ desorption peak and the high temperature $\mathrm{D}$ desorption peak behave independently of each other, indicating that the D desorbing in each peak could be attributed to different defects. Moreover, the shape of each D desorption peak remains largely unaltered with annealing. This similar D desorption spectrum behaviour was also observed in [18].

As we are mainly interested in the densities of the defects that survive the annealing process, we will use a simplified simulation procedure. In this approach both the WD-AD and WDW-AD experimental series will be simulated in the same manner, where the history of the samples with several consecutive W irradiations and D exposures will be disregarded. Instead, the simulation will be run as if the $\mathrm{W}$ samples were $\mathrm{W}$ irradiated only once. The amount of displacement damage that the proxy $\mathrm{W}$ irradiation would cause will be equal to the surviving density of defects after the annealing procedure. Such a proxy pre-damaged sample is then subjected to the same D exposure as was the case in our experiment, which simulates the second D exposure done after annealing, which was used to decorate the surviving defects. In such a way the simplified modelling approach can be condensed into three main steps:

(1) Exposure of a pre-damaged $\mathrm{W}$ sample to $\mathrm{D}$ ions with energy $<5 \mathrm{eV} / \mathrm{D}$ with a flux of $5.6 \times 10^{19} \mathrm{D} / \mathrm{m}^{2}$ s for 72 hours at $370 \mathrm{~K}$, yielding a D fluence of $1.5 \times 10^{25} \mathrm{D} / \mathrm{m}^{2}$.

(2) Cool off to $295 \mathrm{~K}$ in vacuum,

(3) Subsequent TDS measurement with a controlled ramp of $3 \mathrm{~K} / \mathrm{min}$.

Preferably, the entire experimental procedure should be modelled, including several W irradiation steps and the full annealing procedure. Unfortunately, as no macroscopic rate-equation model of defect annealing or evolution exists, this is not possible.

To properly determine the densities of defects and their fill-level de-trapping energies the depth distribution of defects must be chosen correctly. As we saw in the experimental results section the D depth profiles in both experimental series were not entirely flat. This meant that although the defect densities have achieved saturation through most of the damaged layer, saturation has 
apparently not occurred near the surface where the damage dose is more than a factor of two smaller than in the damage peak. Still, in the spirit of a simplified simulation approach, we chose to use a completely flat depth distribution for all of the defects, which can be parametrized as:

$$
n_{i}(x)=n_{i}^{0}\left(1+e^{\frac{x-x_{0}}{\delta x}}\right)^{-1}
$$

Equation 1 is a phenomenological description (based on the Fermi-Dirac distribution) of a flat defect distribution that is flat up to a depth of $x_{0}=2.05 \mu \mathrm{m}$ and reduces to 0 in a transition which is $\delta x=0.06 \mu \mathrm{m}$ wide. The parameters $x_{0}$ and $\delta x$ were determined by fitting the $\mathrm{D}$ depth profiles seen in Fig. 1a-e and Fig. 2a-e. The simulation outputs of the simplified approach are the number of different defects needed to adequately describe the experimental results and their surviving densities after annealing $\left(n_{i}^{0}\right)$ as well as the de-trapping energies $\left(E_{i}^{j}\right)$ of their fill-levels. The index $i$ denotes the defect type and index $j$ denotes the number of the fill-level.

Also the boundary conditions for the solute D at the $\mathrm{W}$ sample surface must be considered when talking about our choice of modelling procedure. Usually with energetic D ions that are implanted directly into the $\mathrm{W}$ bulk, we can assume that the time-scales for diffusion from deep in the bulk to the surface are much larger than the time-scale for D desorption from the surface. This further means we can assume that a deuterium atom that comes to the surface of the material from the bulk desorbs immediately [25]. For this reason, we use the Dirichlet boundary condition at the surface (solute concentration at the surface is equal to 0 ).

As the D ions are implanted directly into the bulk, the implantation flux is modelled as a volume source of solute D in the bulk. The distribution of the source is calculated with SRIM and is modelled as a Gaussian distribution with a projected mean range of $0.7 \mathrm{~nm}$ and a lateral dispersion of $0.5 \mathrm{~nm}$ for the $5 \mathrm{eV} / \mathrm{D}$ plasma exposure. The coefficient of reflection for the $\mathrm{D}$ ions hitting the sample surface used in our experiment was 0.5 [26].

\section{Simulation Results}

The first thing we can learn by modelling the D desorption spectra shown in Fig. 1f and Fig. $2 \mathrm{f}$ is that the best fits are achieved when three distinct defect types with several fill-levels are used. The de-trapping energies of the observed defects are summarized here: 
- defect I has five fill-levels with de-trapping energies $(1.08 \pm 0.03,1.16 \pm 0.02,1.24 \pm$ $0.03,1.34 \pm 0.02,1.46 \pm 0.03) \mathrm{eV}$,

- defect II has two fill-levels $(1.68 \pm 0.02,1.86 \pm 0.03) \mathrm{eV}$ and

- defect III has one fill-level $(2.05 \pm 0.01) \mathrm{eV}$.

These de-trapping energies are associated with a diffusion barrier of $0.2 \mathrm{eV}$ [27], a diffusion preexponential coefficient of $1.9 \times 10^{-7} \mathrm{~m}^{2} \mathrm{~s}^{-1}$ [27] and a de-trapping attempt frequency of $10^{13} \mathrm{~s}^{-1}$ [27]. The errors for determining the de-trapping energies are derived from the scatter of the de-trapping energies used for all of the fits. The energy errors translate into a temperature error of around $20 \mathrm{~K}$.

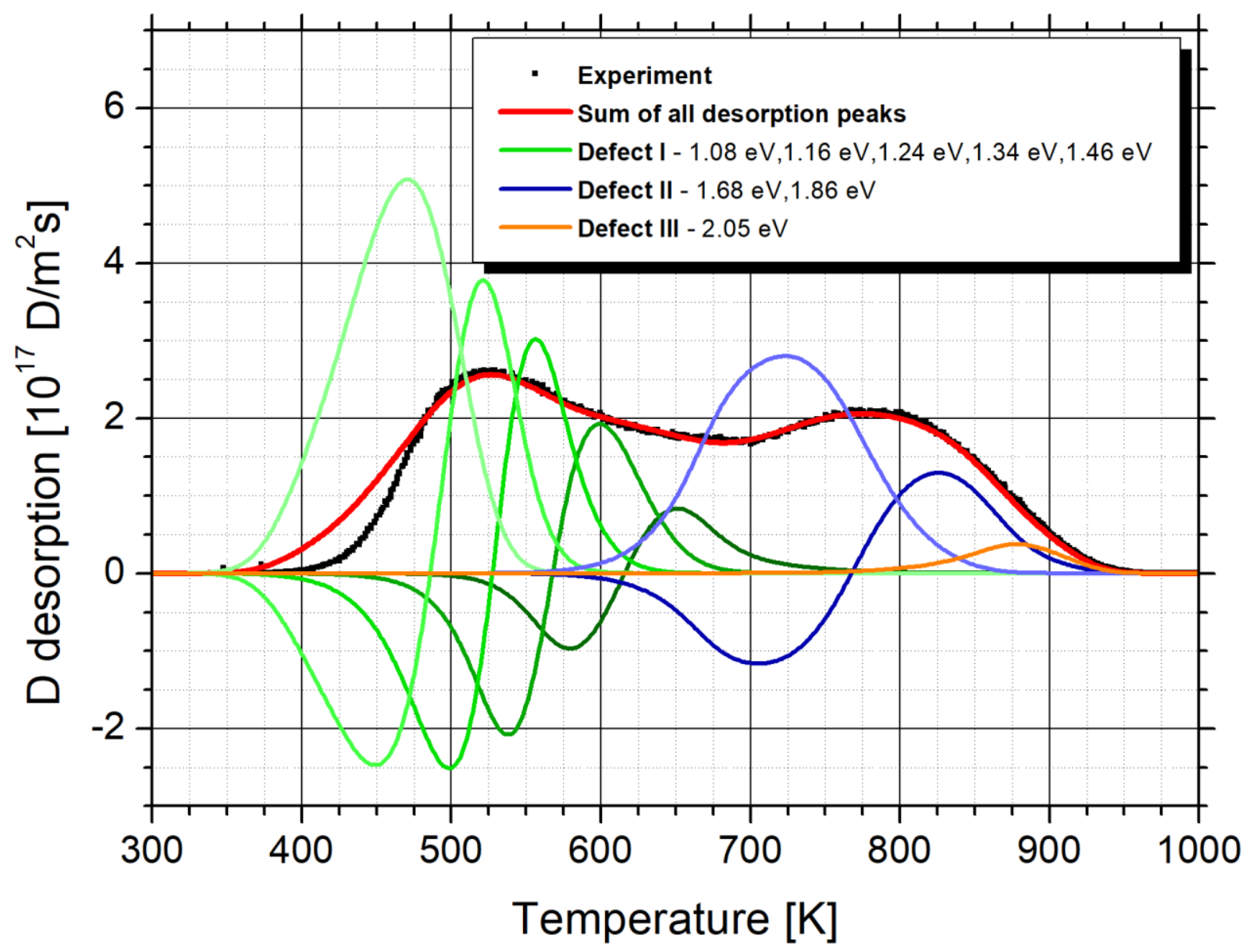

Figure 5: The comparison between the experiment and simulation of the D desorption spectrum of the sample annealed at $600 \mathrm{~K}$, which is part of the WD-AD experiment. The experimental results are plotted as black squares, desorption peaks from fill-levels of defect I are plotted as green lines, from defect II as blue lines and from defect III as the orange line. The sum of all desorption peaks from the simulation is plotted as the red line.

The summary of the observed defects is also shown in Fig. 5 where the simulated D desorption spectrum (red line) is shown as a sum of D desorption contributions from individual fill-levels and 
is compared to the experimental D desorption spectrum of the WD-AD sample annealed at $600 \mathrm{~K}$ (black dots). The $\mathrm{D}$ desorption contributions shown in Fig. 5 are color-coded in a way that D desorption from defect I is plotted in shades of green, from defect II with shades of blue and from defect III with orange. As can be observed in Fig. 5 defect type I is responsible for D desorption in the $370-700 \mathrm{~K}$ range, defect type II in the 600-900 K range and defect type III in the $820-950 \mathrm{~K}$ range. Desorption of D in Fig. 5 is negative for some fill-levels. This is a feature of the fill-level picture of HI trapping and comes from the fact that as fill level $i+1$ is de-trapping, fill level $i$ is filling up. When drawing a $\mathrm{D}$ desorption spectra desorption from a trap is defined as positive, which means that the filling of the lower fill level $i$ is counted as negative.

Now let us continue with comparing the modelling and the experimental results that are seen in Figs. 6a-e. The experimental results are plotted with black (WD-AD) and grey (WDW-AD) connected dots, while the modelling results are drawn with red (WD-AD) and pink (WDW-AD) lines.
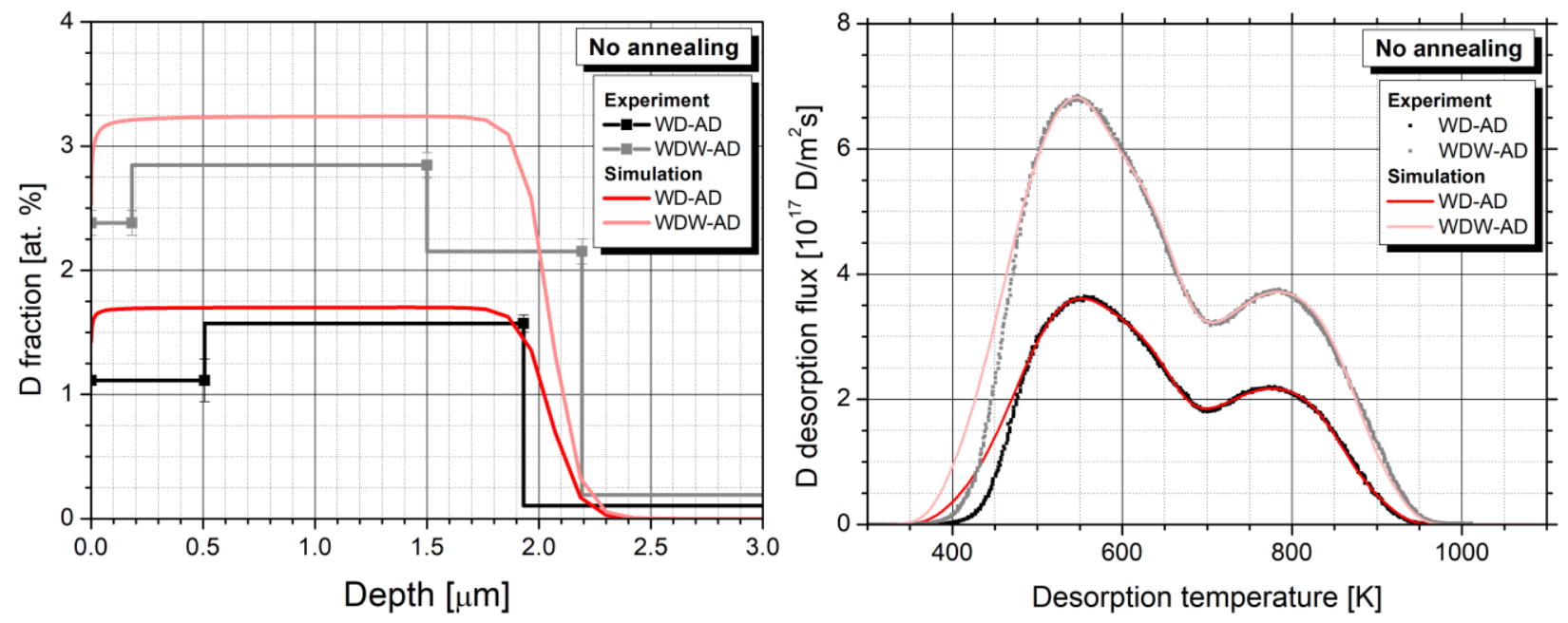

(a) 

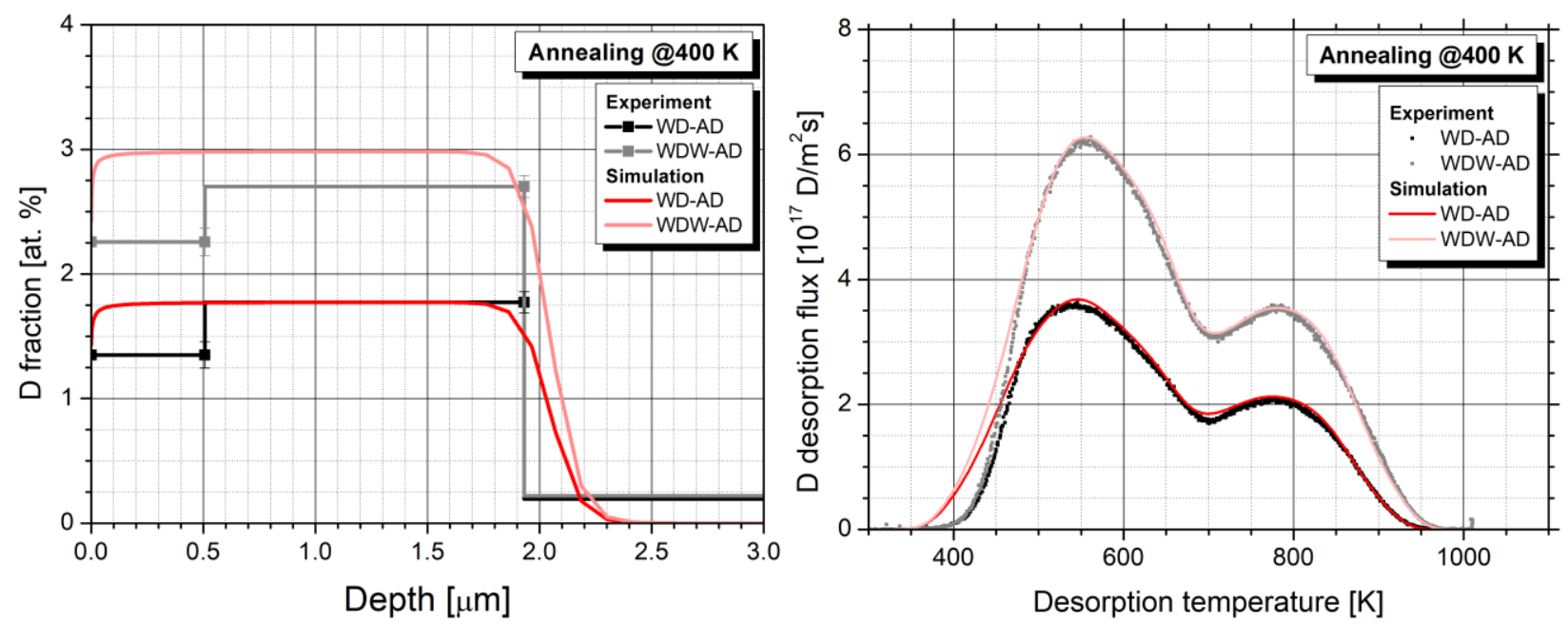

(b)
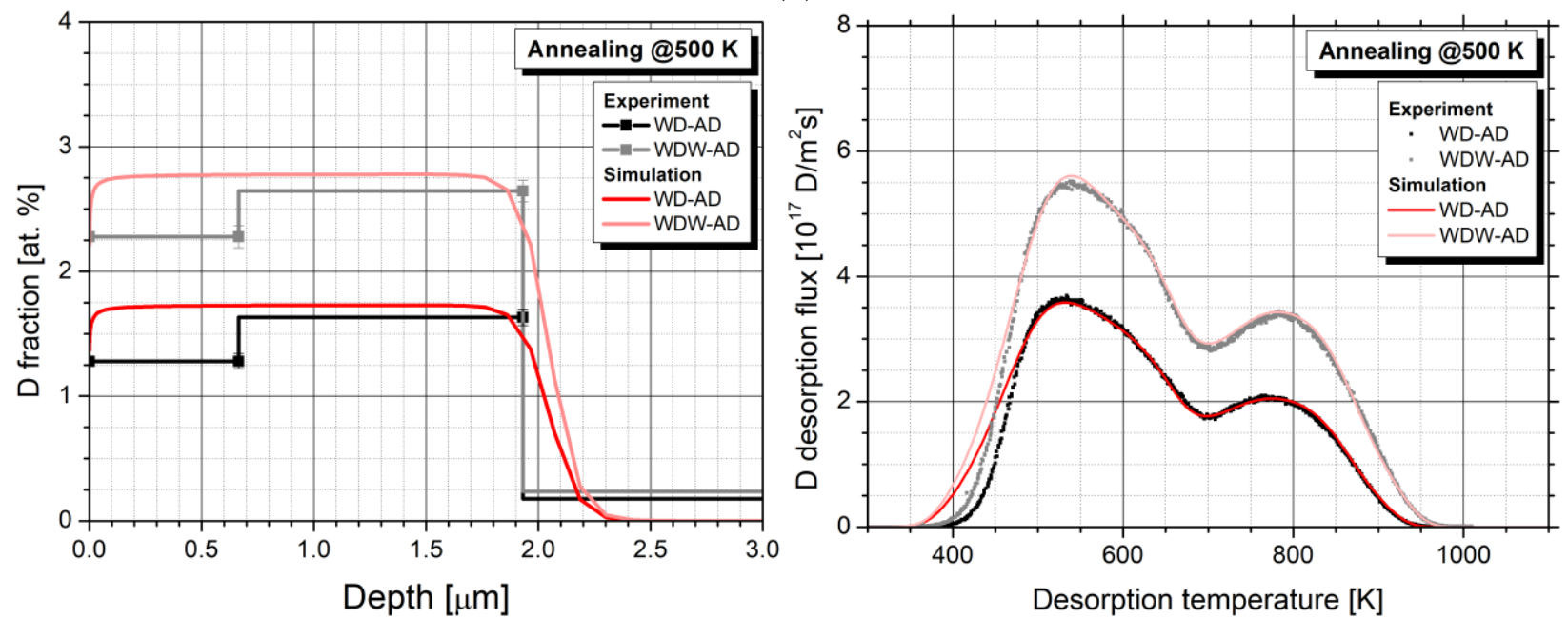

(c)
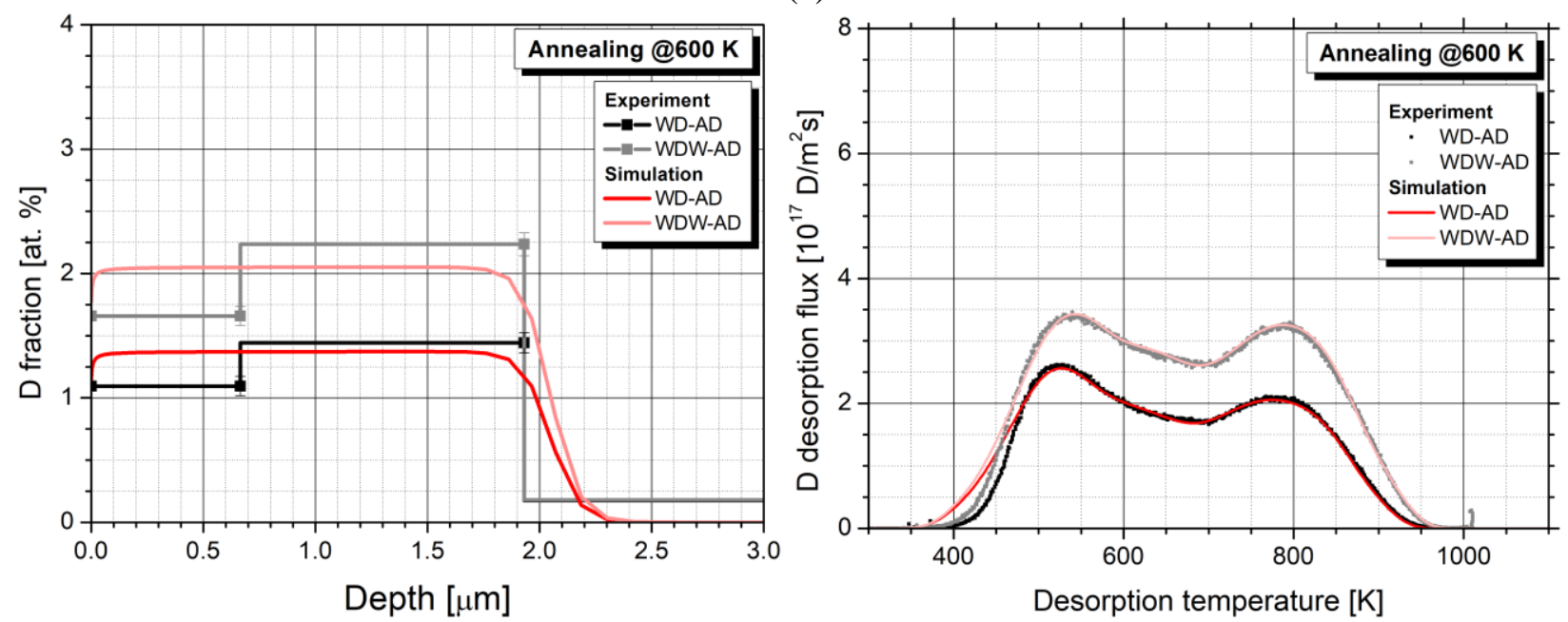

(d) 


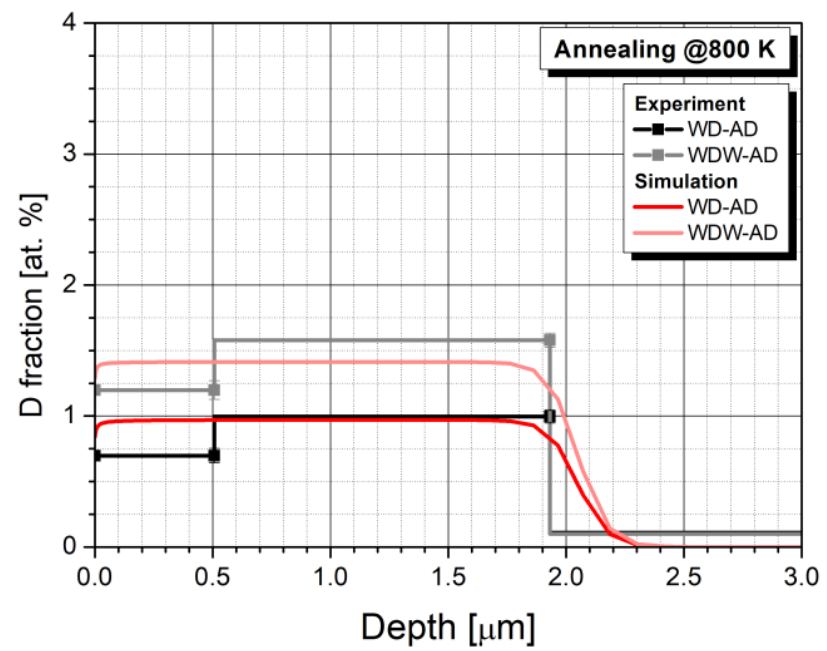

Depth $[\mu \mathrm{m}]$

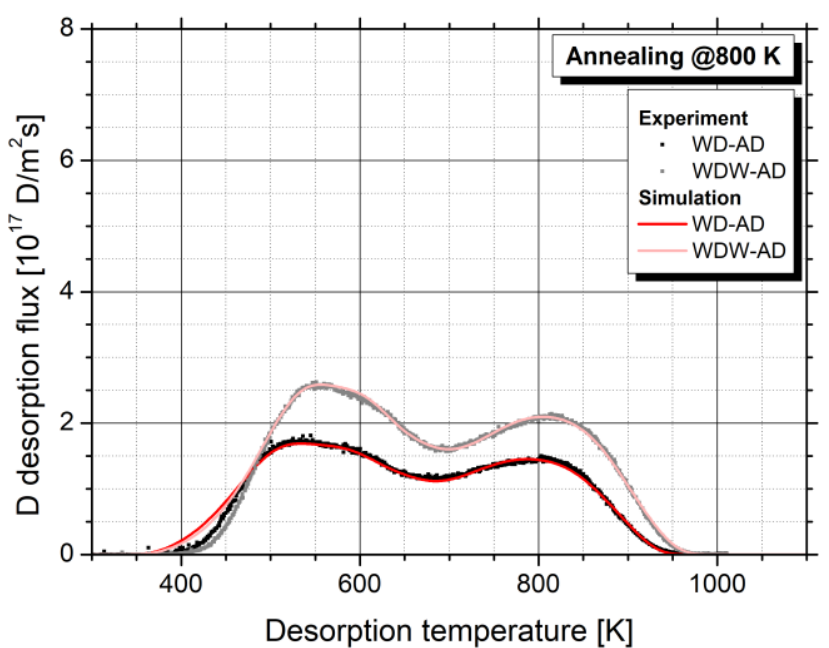

(e)

Figure 6: Comparison of the experimental data and the respective fits using the MHIMS-R code. The experimental data are shown in black (WD-AD) and grey (WDW-AD) while the simulation results are plotted with red (WD-AD) and pink (WDW-AD).

We can see that the maximum D concentration in most of the D depth profiles are well reproduced, except in the case of the reference double-irradiated sample in Fig. 6a and the double-irradiated sample annealed at $400 \mathrm{~K}$ as seen in Fig. 6b. This can be attributed to the fact that a low temperature D desorption early rise can be seen in all of the simulation D desorption spectra which does not exist in the experimental results as can be seen in the D desorption spectra in Figs. 6a-e. This rise accounts for approximately a $10 \%$ overestimation of the total $\mathrm{D}$ retention and therefore also D maximum concentration. This overestimation is most pronounced in the WDW-AD reference sample and in the sample annealed at $400 \mathrm{~K}$, because the match between the total D retention derived from the NRA and TDS measurements is the closest in these two cases (see Fig. 4a). In other cases, the D retention measured by TDS is approximately $10 \%$ less than the D retention measured by NRA, which coincidentally compensates the $10 \%$ simulation overestimation because of the low temperature D desorption early rise. This rise is an unexplained but common occurrence when the simulated D desorption spectra are compared to experimental spectra [18], [25], [28], [29] and we have not been able to eliminate it. Otherwise, the D desorption spectra are fitted very well. The structure of two peaks centered at $520 \mathrm{~K}$ and $780 \mathrm{~K}$ and a high-temperature $\mathrm{D}$ desorption tail are well reproduced by the simulation with our choice of fitting parameters. To determine the quality of the fits the reduced chi square is used as a figure of merit. It is defined as: 


$$
\chi_{\text {red }}^{2}=\frac{1}{N-1} \sum_{i=1}^{N} \frac{\left(\Gamma_{i}^{\text {sim }}-\Gamma_{i}^{e x p}\right)^{2}}{\sigma_{i}^{2}}
$$

$\mathrm{N}$ is the number of points in the experimental desorption spectra. $\Gamma_{i}^{e x p}$ is the experimental desorption flux at a temperature $\mathrm{T}_{\mathrm{i}}$ and similarly $\Gamma_{i}^{\text {sim }}$ is the simulation desorption flux at $\mathrm{T}_{\mathrm{i} .} \sigma_{\mathrm{i}}$ is the experimental error of $\mathrm{D}$ desorption at temperature $\mathrm{T}_{\mathrm{i}}$. It was determined directly from the $\mathrm{D}$ desorption measurement count rates, assuming that the relative error follows Poisson statistics $\sigma_{\mathrm{i}}$ $=\left(\Gamma_{\mathrm{i}}^{\exp }(\text { count })\right)^{-1 / 2}$. This in combination with the intrinsic error tied to quadrupole signal stability (see section 3.3.) amounts to the total experimental error that we use in the reduced chi square calculation. The $\chi_{\text {red }}^{2}$ calculation was limited to TDS temperatures between 470 and $900 \mathrm{~K}$ where $90 \%$ of retained $\mathrm{D}$ was desorbed, due to the existence of the low-temperature D desorption early rise. The calculated $\chi_{\text {red }}^{2}$ are listed in Table 1 for each desorption spectrum. Their values are close to 1 which confirms the quality of the fit.

The densities of the three defect types derived from the modelling are plotted in Fig. 7a and Fig. $7 \mathrm{~b}$ as a function of annealing temperature. The densities of the defects are explicitly written out in Table 1 for all annealing temperatures. The annealing behaviour is specific to the 2 hour annealing procedure. Concentrating on the simulation results for the WD-AD experimental series plotted in Fig. 7a, we can see that the density of defect type I, that desorbs in the D desorption peak centered at $520 \mathrm{~K}$, shows no decrease due to annealing for temperatures lower or equal to $500 \mathrm{~K}$. At an annealing temperature of $600 \mathrm{~K}$ about $30 \%$ of its reference density is lost and at $800 \mathrm{~K}$, approximately $50 \%$ of the reference amount is annealed. In our previous publication [18] this defect was inferred to be single vacancies. Defect type II, that desorbs in the high-temperature D desorption peak centered at $780 \mathrm{~K}$, shows no reduction in density for temperatures lower or equal to $600 \mathrm{~K}$, while at $800 \mathrm{~K}$ around $33 \%$ of reference defect density is annealed. Defect type II was inferred to be small vacancy clusters in Ref. [18]. Defect type III shows no behaviour with rising annealing temperature. The third defect was inferred to be large vacancy clusters in Ref. [18].

When considering the simulation results of the WDW-AD experimental series a very important conclusion can be made. As we have shown, the experimental results of the WDW-AD experimental series could be successfully replicated by the exact same choice of fitting parameters as the fitting parameters used to replicate the WD-AD experimental series. More specifically we 
have used the same number of distinct defect types, the same number of fill-levels for each defect type and the same de-trapping energies for each fill-level. This means that the D presence during the second $\mathrm{W}$ irradiation only created additional defects of the already existing defect types. No new defect types were created. This fact is further corroborated by the fact that the same annealing temperature thresholds and similar annealing temperature behaviour was observed in both WDAD and WDW-AD experimental series. Some small reduction in the density of the defects I and II can be observed even below their respective annealing temperature thresholds these reductions are within the experimental uncertainties and are not considered significant.

Table 1: Defect densities remaining after the annealing of the sample at various temperatures for 2 hours are given for both experimental series. Also shown are the reduced chi square values for the fits of the D desorption spectra for all samples.

\begin{tabular}{|c|c|c|c|c|c|c|c|c|}
\hline \multirow[b]{2}{*}{$\mathbf{T}_{\text {anneal }}[\mathbf{K}]$} & \multicolumn{3}{|c|}{ WD-AD series } & & \multicolumn{3}{|c|}{ WDW-AD series } & \\
\hline & $\begin{array}{c}\text { Defect I } \\
\text { [at. \%] }\end{array}$ & $\begin{array}{c}\text { Defect II } \\
\text { [at. \%] }\end{array}$ & $\begin{array}{c}\text { Defect III } \\
\text { [at. \%] }\end{array}$ & $\chi_{\text {red }}^{2}$ & $\begin{array}{c}\text { Defect I } \\
\text { [at. \%] }\end{array}$ & $\begin{array}{c}\text { Defect II } \\
\text { [at. \%] }\end{array}$ & $\begin{array}{c}\text { Defect III } \\
\text { [at. \%] }\end{array}$ & $\chi_{\text {red }}^{2}$ \\
\hline 370 & 0.215 & 0.290 & 0.050 & 0.88 & 0.426 & 0.520 & 0.080 & 0.92 \\
\hline 400 & 0.230 & 0.290 & 0.050 & 0.57 & 0.382 & 0.495 & 0.090 & 1.26 \\
\hline 500 & 0.225 & 0.280 & 0.050 & 0.99 & 0.345 & 0.480 & 0.090 & 0.81 \\
\hline 600 & 0.153 & 0.280 & 0.050 & 0.92 & 0.215 & 0.440 & 0.100 & 0.46 \\
\hline 800 & 0.107 & 0.189 & 0.060 & 0.51 & 0.155 & 0.290 & 0.060 & 0.82 \\
\hline
\end{tabular}

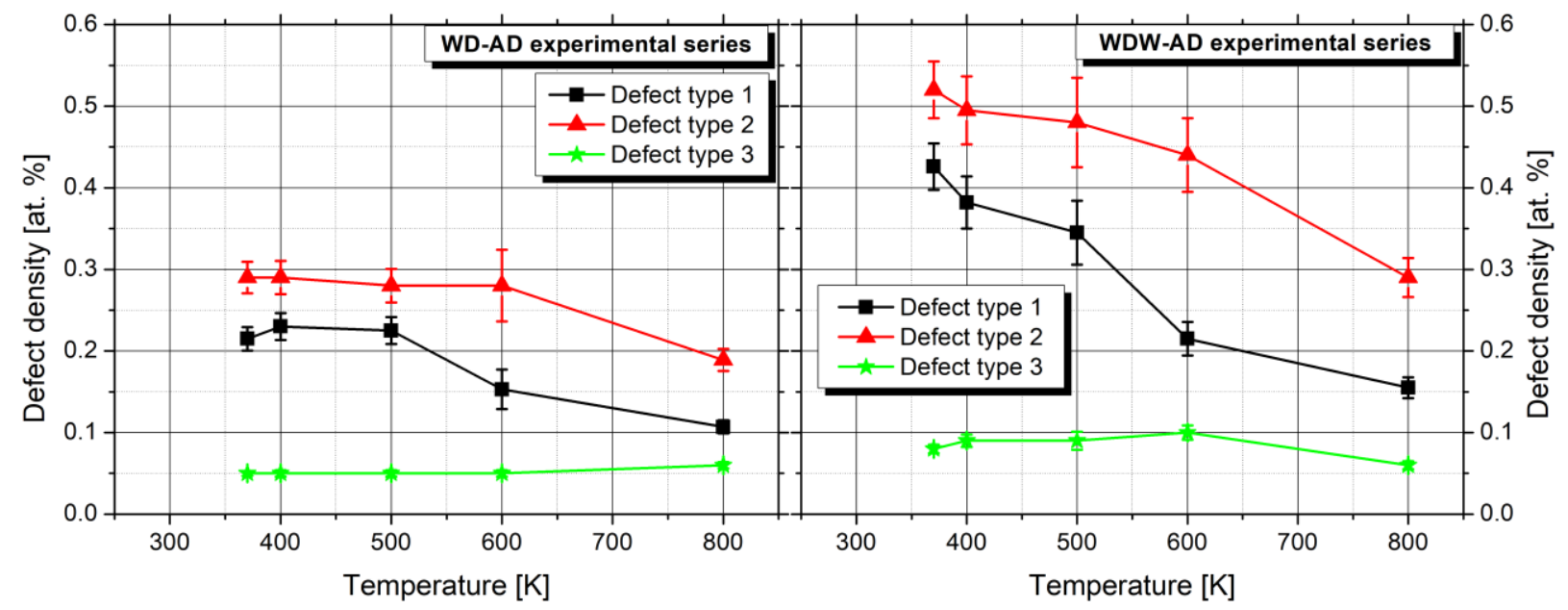

(a)

(b)

Figure 7: The defect densities that survive the annealing process at a specific temperature are shown. Defect I identified as a vacancy is plotted with black squares, defect II identified as a small vacancy clusters with red triangles and defect III identified as large vacancy clusters/voids is plotted with green stars. 
With the knowledge we gained by simulating the $\mathrm{D}$ depth profiles and $\mathrm{D}$ desorption spectra at the end of the experimental procedure we can also simulate the $\mathrm{D}$ desorption during the ramp and hold part of the annealing procedure. To simulate the procedure, no fitting was done. The de-trapping energies are exactly the same as specified in the simulation section but we must still choose which defect density we shall use as no defect evolution due to annealing is implemented in MHIMS-R. We expect that $\mathrm{D}$ desorption is much faster than defect evolution, therefore we choose to use the defect densities of the reference sample to simulate the ramp and hold procedure for all samples. The simulation results are shown in Fig. $8 \mathrm{a}$ and $8 \mathrm{~b}$. The simulation of the D desorption during annealing was performed with the actual heating ramp of the sample, being $24 \mathrm{~K} / \mathrm{min}$. The simulation line is a weighted sum of $\mathrm{D}$ desorption from a once- and twice-irradiated $\mathrm{W}$ samples. The weights are proportional to the surface area of the once and twice-irradiated part of the sample. In this specific case we assume that exactly half of each sample was irradiated twice.

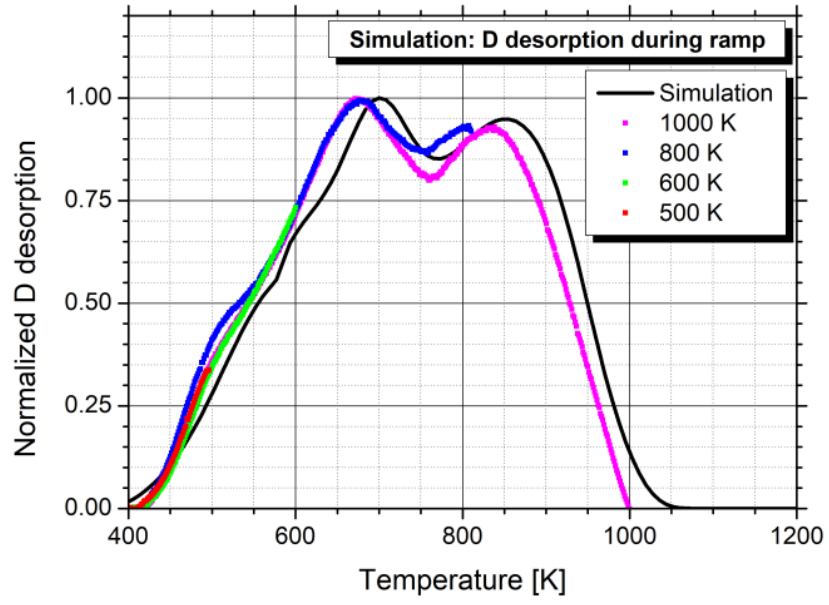

(a)

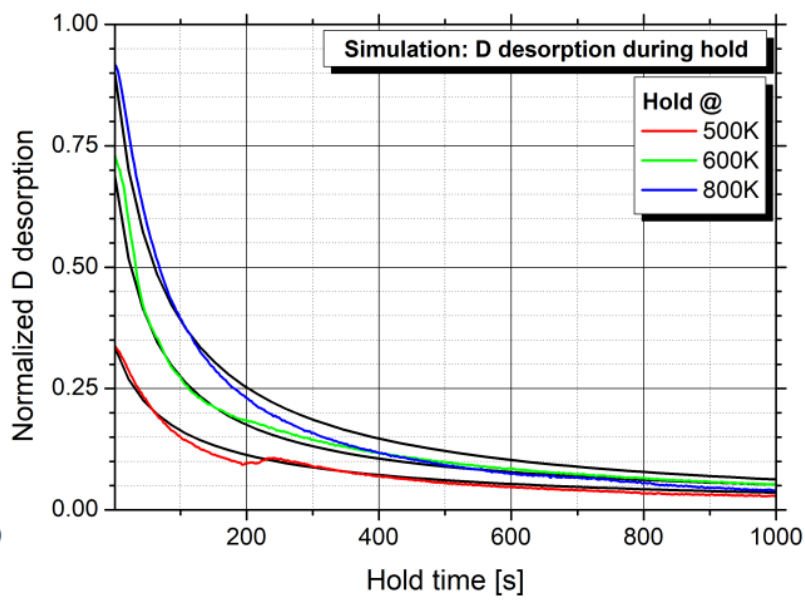

(b)

Figure 8: The simulation and experimental comparison of the D desorption during the ramp (24 K/min temperature ramp) and hold (annealing) procedure.

We see that the D desorption during the ramp as shown in Fig. 8a is replicated reasonably well by the simulation. The reduction of $\mathrm{D}$ desorption in the low temperature desorption peak and the proportional increase in the high-temperature desorption peak is replicated very well. The simulation only fails to reproduce the exact desorption peak temperatures as it overestimates them by approximately $50 \mathrm{~K}$. The exact reason for this is unknown but both experimental and simulation reasons could be at play. From the viewpoint of the simulation the fit could have easily been 
improved by slightly changing the de-trapping energies as was done to fit the other experimental results. We chose not to do this as not much additional value would be gained as the fit is already satisfactory. The D desorption during the hold part as shown in Fig. $8 \mathrm{~b}$ is also well replicated.

\section{Discussion}

By correlating the annealing behaviour of the different defect types with findings in the literature we can try to identify the specific defect types. Previous experiments, modelling and $a b$ initio calculations have reported de-trapping energies similar to the ones we have used, of which a summary has been made in Ref. [18]. We must stress that in Ref. [18] it was not our intention to pinpoint the exact de-trapping energies of fill-levels for the defects used in the simulation. Instead, the defect types were inferred by comparing the derived de-trapping energies with theoretical results available in the literature, where the literature data was interpreted as broad de-trapping energy bands instead of exact values. In this work, we will merely compare the expected annealing behaviour based on the inferred defect types, with the behaviour we observed in the experimental and simulation results.

We also wish to stress that according to various theoretical calculations for vacancies [27], [30], [31] and vacancy clusters [32] many more fill-levels with lower de-trapping energies are available to trap D for our exposure conditions. By including more fill-levels into our simulation the number of free simulation parameters would increase drastically. As there would be no way to adequately validate the values of all of the free parameters used for such a simulation, we have decided to only use the minimum free parameter set which recreates the experimental results. If more fill-levels would be added, this would most certainly affect the exact values of defect densities and de-trapping energies we have reported in this work, but the general message this work tries to convey would remain unchanged.

\subsection{Defect type I - vacancies}

Defect I, drawn with black squares in Fig. 7a and 7b, starts to anneal in the 500 to $600 \mathrm{~K}$ temperature range, while at lower annealing temperature no behaviour is observed as the density is constant. The start of vacancy mobility and clustering has been observed by the positron annihilation technique [33]-[35] in the same temperature range. This is often called recovery stage III and has been linked with vacancy evolution through clustering mechanisms. This is in line with 
our identification of defect type I as a single vacancy and is consistent with the summary given in [18].

\subsection{Defect type II - small vacancy clusters}

The density of defect type II is constant in the 300-600 K annealing temperature range, and annealing is only observed in the 600-800 K range. As defect type II is assumed to be small vacancy clusters the annealing mechanism is expected to be cluster dissociation [36].

We assume that the rate of dissociation can be written as an Arrhenius like term [36] similar to D de-trapping from defects:

$$
R_{\text {diss }}^{n}=v_{0} \exp \left(-\frac{E_{\text {bind }}^{n}+E_{\text {diff }}^{v a c}}{k_{B} T}\right)
$$

Here $R_{\text {diss }}^{n}$ is the Arrhenius dissociation rate, $v_{0}$ is the attempt frequency of a vacancy trying to escape a cluster through dissociation. We assume this dissociation frequency to be the same as the attempt frequency for vacancy diffusion equal to $v_{0}=5.5 \times 10^{12} \mathrm{~s}^{-1}[37]$. $E_{\text {diff }}^{\text {vac }}=1.7 \mathrm{eV}$ is the activation energy associated with vacancy diffusion [38] and $E_{\text {bind }}^{n}$ is the binding energy of a vacancy to a n-sized vacancy cluster. Quite some work has already been devoted to the binding energies of vacancies to vacancy clusters by ab initio Density Functional Theory (DFT) and Embedded Atom Method (EAM) calculations [38]-[43]. The vacancy binding energy to the cluster with a specific size as derived from these studies is plotted in Fig. 9a. The scatter of the data is quite large, which is why we have calculated the mean and the standard deviation of the binding energies as a function of cluster size for clusters smaller than 15 vacancies. This is shown in Fig. $9 \mathrm{~b}$ drawn as black squares. For larger vacancies only one data set is available, making any interpretation unreliable. From the means and standard deviation, we have calculated an analytical fit with a reduced chi square of 0.76 which can be written as:

$$
E_{\text {bind }}^{n}=E_{0} \sqrt{n-2.8}
$$

Here $\mathrm{E}_{0}=0.5 \mathrm{eV}$ is the fit parameter which produced the best fit value and $n$ is the number of bound vacancies. The analytical fit is plotted in Fig. $9 \mathrm{~b}$ as a red line alongside the mean and 
standard deviation which are plotted in black. Of course, our analytical fit is only a crude approximation, but until more consistent data is available this is the only course forward.

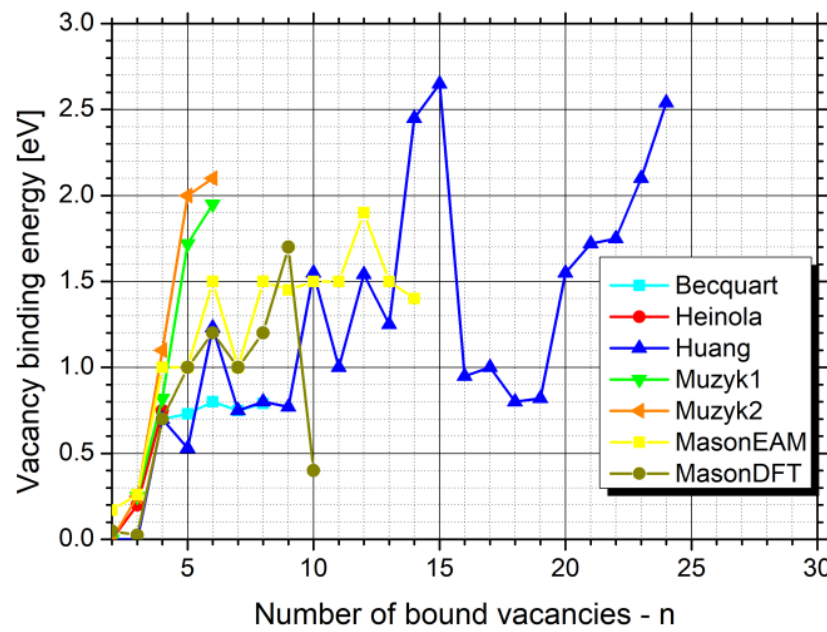

(a)

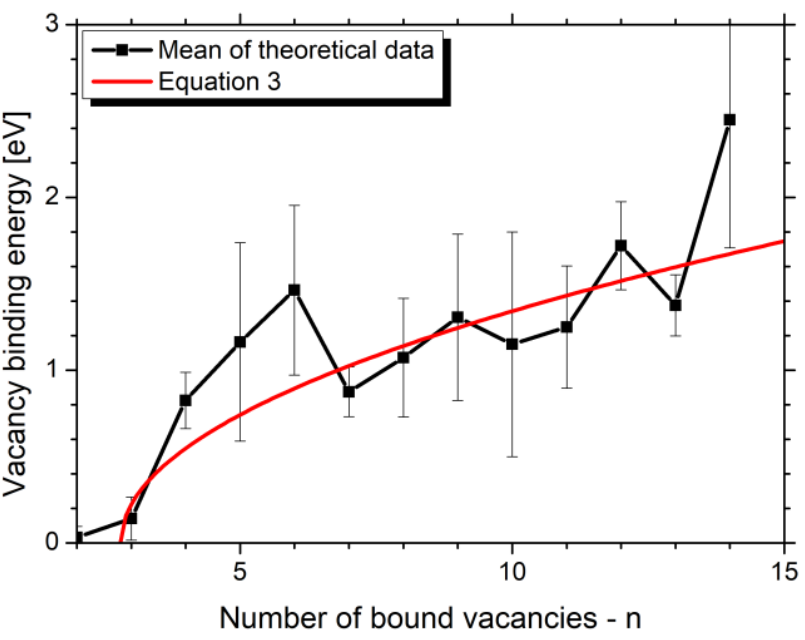

(b)

Figure 9: (a) Single vacancy binding energy to a vacancy cluster as a function of total bound vacancies based on various atomistic studies. (b) As the data scatter of vacancy binding energies is very large as can be seen in (a) we have calculated the mean vacancy binding energy at each number of bound vacancies, which is plotted with black squares. An analytical fit with equation 2 is shown in red.

As the annealing in our experiments is done at a constant temperature $T_{\text {anneal }}$, the density of vacancy clusters decreases with time following an exponential decay law according to equation (3). In both the WD-AD and WDW-AD annealing experiment about one third of the created small vacancy clusters were annealed at an annealing temperature of $T_{\text {anneal }}=800 \mathrm{~K}$, which means we can write:

$$
N\left(t_{\text {anneal }}\right)=\frac{2}{3} N_{0}=N_{0} \exp \left(-R_{\text {diss }}^{n} t_{\text {anneal }}\right)
$$

The dissociation rate can be expressed as $R_{\text {diss }}^{n} t_{\text {anneal }}=\ln (3 / 2)$. By inputting the dissociation rate defined in equation (3) into the equation (5) which is bound by our experimental results we can calculate the binding energy of a vacancy cluster with size $n$ where a third of its initial defect concentration would dissociate at a temperature $T_{\text {anneal }}$ with the following equation:

$$
E_{\text {bind }}^{n}=k_{B} T_{\text {anneal }} \ln \left(\frac{v_{0} t_{\text {anneal }}}{\ln \left(\frac{3}{2}\right)}\right)-E_{\text {diff }}^{v a c}=0.85 \mathrm{eV}
$$


This binding energy, according to equation (4) corresponds to a vacancy cluster of size $n=5.8$. Of course sizes of vacancy clusters are integers, but this calculated size just means that the majority of cluster dissociation that explains our drop in small vacancy cluster annealing at $800 \mathrm{~K}$ should be mainly due to dissociation of cluster with sizes 6 and a smaller contribution by dissociation of clusters with 5 captured vacancies. Larger clusters should be stable at $800 \mathrm{~K}$ for two hours of annealing.

\subsection{Defect type III - large vacancy clusters}

From Fig. 7a and $7 \mathrm{~b}$ we can see that the density of defect III is constant in the entire temperature range. This is again consistent with our assumption made in Ref. [18] that these are large vacancy clusters. Vacancy clusters, according to equation (2), should have binding energies of around $2.07 \mathrm{eV}$ for 20 captured vacancies and $2.61 \mathrm{eV}$ for 30 captured vacancies. According to these binding energies their annealing should start somewhere around 1150-1250 K, which is well beyond the temperature range of our experiment.

\subsection{Mechanisms of displacement damage evolution}

We have highlighted some of the possible mechanisms of the vacancy-type defect evolution that can describe the annealing behaviour observed in the experimental and simulation results. Did not yet address various self-interstitial atom (SIA) structures, such as dislocation lines and loops. Their de-trapping energy is most likely too low for the $\mathrm{D}$ to be trapped in them for our exposure conditions [44]. In principle, SIA dislocation structures could also possibly affect the annealing behaviour of vacancy-type defects. However, this is unlikely as because most of the SIA atoms are lost to various free surfaces such as grain boundaries very quickly after the process of W irradiation because of their high mobility [45]-[48]. In samples which were treated in a manner similar to ours, the volumetric density of dislocations has been measured to be approximately $10^{-4}$ at.\% [9], [49]. Assuming all of the dislocations measured are of the SIA type, the measured density of dislocations provides an upper limit on the density of all SIAs that are available during our annealing process. Considering that the densities of available SIAs is orders of magnitude smaller than the densities of vacancy-type defects determined by our simulation, any interaction between

SIAs and other vacancy-type defects should be much weaker compared to vacancy mobility and subsequent vacancy clustering. Based on the densities of dislocation structures alone, one would 
also not except them to contribute significantly to D retention at the exposure conditions used in our experiment.

If we assume that vacancy mobility, clustering and cluster dissociation are the main drivers of defect evolution the behaviour of the different defect types observed in Table 1 and Fig. 7 might be slightly confusing. One would expect that as a single-vacancy is captured in a cluster in the 500$600 \mathrm{~K}$ range, the density of vacancy clusters would rise for the same value that the density of singlevacancies fell. However, our results become clearer as one considers what happens with the density of traps sites instead, as this is the relevant quantity when D trapping is simulated using macroscopic rate equation codes such as MHIMS-R. As a single-vacancy is captured by a cluster with $N$ bound vacancies, the cluster transforms into a cluster with $N+1$ bound vacancies. In doing so, one trap site for $\mathrm{D}$ is lost in the form of the single vacancy trap site and no new trap sites become available as the number of D a cluster can capture is only weakly dependant on cluster size [32]. Such reasoning is in line with the behaviour of densities of defects that we have observed in Table 1 and Fig. 7.

\subsection{Effect of $D$ on displacement damage evolution}

We have experimentally shown in Fig. 3b that D desorption happens much faster than the defect evolution process is expected to occur. This means that during the majority of the 2-hour annealing hold the defects evolve with a steady-state trapped D concentration. To fully determine the effect of $\mathrm{D}$ on defect evolution it is important to determine what the steady-state trapped $\mathrm{D}$ concentrations are for each defect type. Therefore, we have plotted this information for both the WD-AD and WDW-AD experimental series in Fig. 10a-e.

It is difficult to correlate the specific occupancies of defects to their annealing behaviour. Therefore, we resort to correlating the defect annealing behaviour to the information if they are completely empty (shown as black) or if they contain at least one D (sum of other colours).

For the WD-AD experimental series a significant portion of single-vacancies (Fig. 10a) becomes empty only at annealing temperatures above $600 \mathrm{~K}$. Small vacancy clusters (Fig. 10c) contain at least one D up to an annealing temperature of $600 \mathrm{~K}$, while at $800 \mathrm{~K}$ the majority of them becomes empty. Similar behaviour is observed also for the large vacancy clusters (Fig. 10e), however only about $50 \%$ of them are empty after annealing at $800 \mathrm{~K}$. 
For the WDW-AD experimental series, similar behaviour is observed with the slight difference that a small portion of all defect types is empty after annealing at $400 \mathrm{~K}$. This is because during the second $\mathrm{W}$ irradiation, the irradiating $\mathrm{W}$ ions create additional damage which is initially empty of D. While some of the D is kinetically de-trapped from the pre-existing defects by the irradiating $\mathrm{W}$ ions [7], [18] and can therefore be re-trapped in the newly formed defects, this process is not efficient enough for our simulation parameters [18] to populate all of the newly created defects with at least one D. Additionally, when the sample is annealed at $400 \mathrm{~K}$ the temperature is not high enough for the $\mathrm{D}$ to be redistributed from the pre-existing defects to the new defects as the majority of $\mathrm{D}$ is too tightly bound. This redistribution can occur only at higher temperature which is why the occupancy behaviour of both experimental series is very similar for annealing temperatures above $500 \mathrm{~K}$. Keep in mind, that all of the reported behaviour is specific to a $24 \mathrm{~K} / \mathrm{min}$ temperature ramp and a 2-hour hold at the specific annealing temperature. 


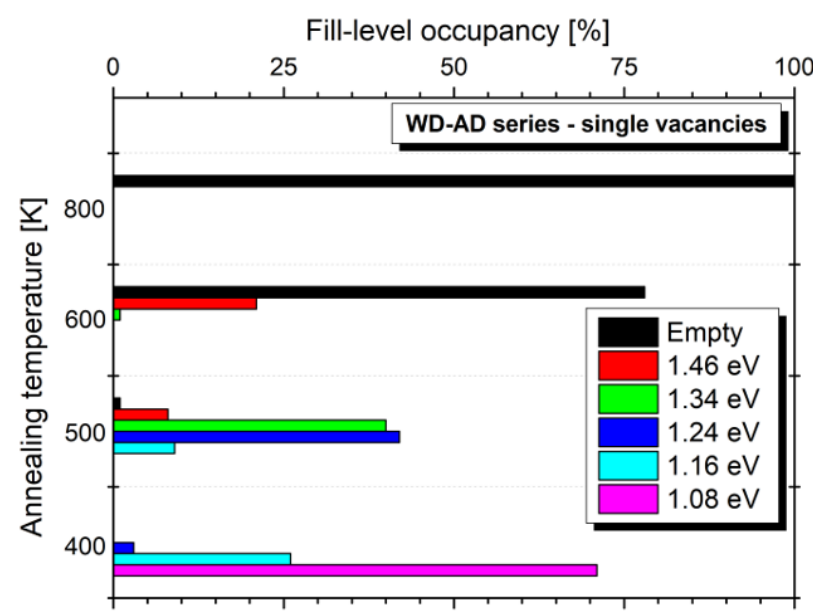

(a)

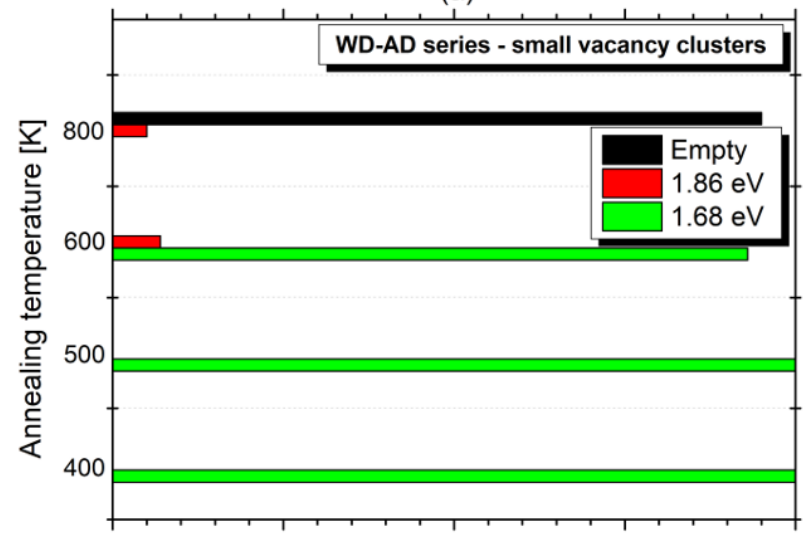

(c)

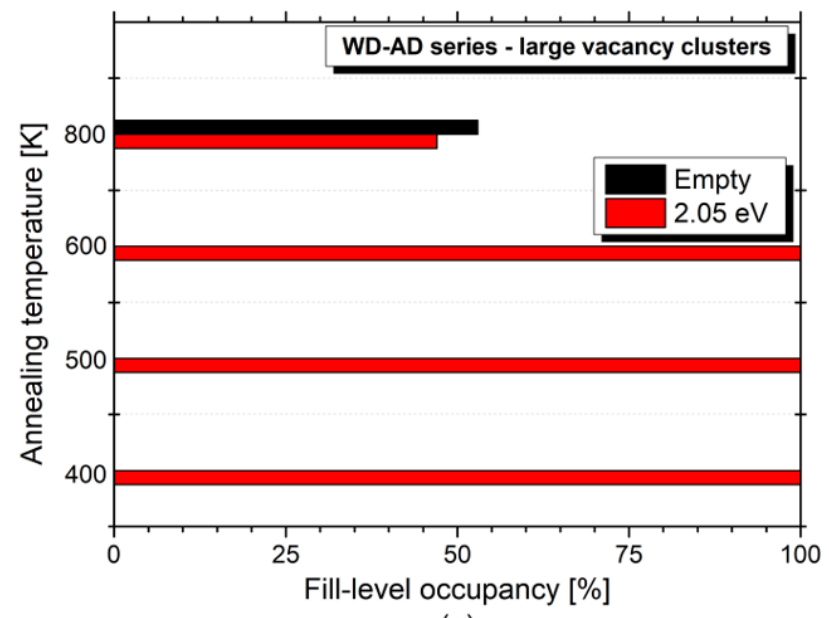

(e)

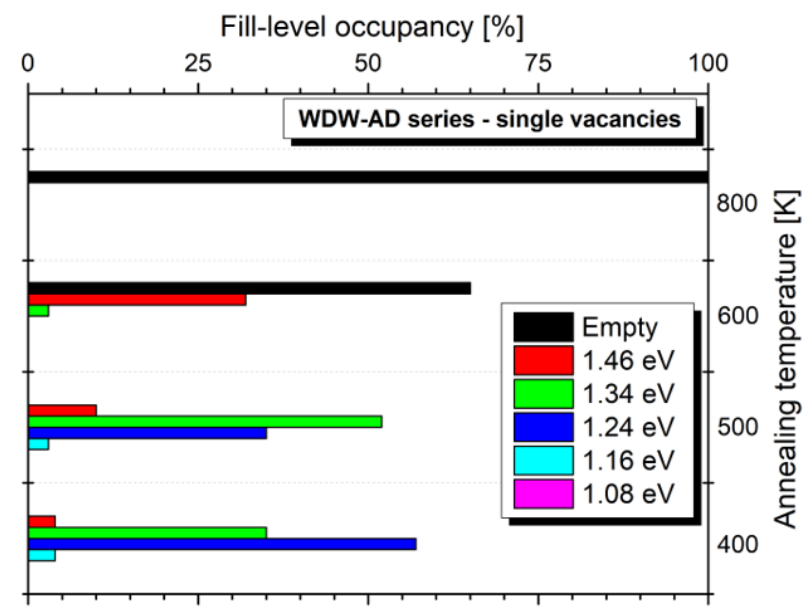

(b)

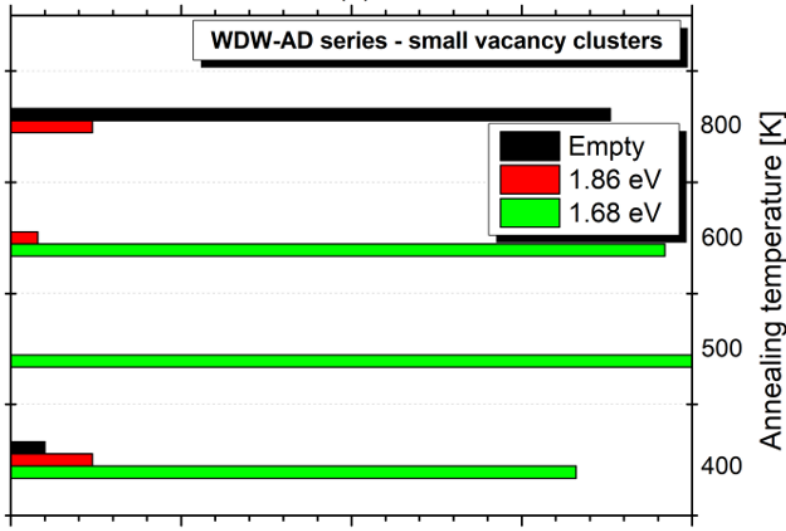

(d)

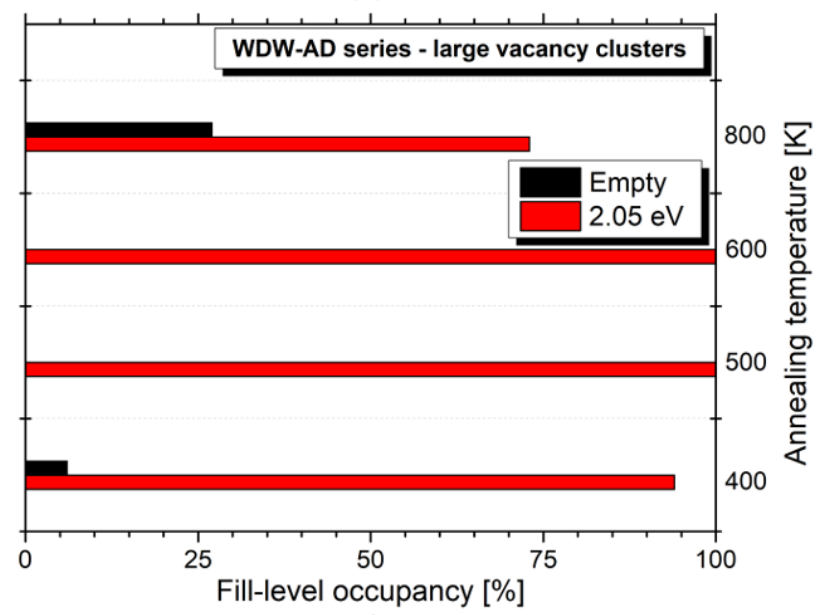

(f)

Figure 10: The occupancy ratios of the different defect type fill-levels after the 2-hour annealing holds at different temperatures are shown. Figures $(a),(c)$ and $(e)$ show occupancy ratios of the WD-AD experimental series, while figures $(b),(d)$ and $(f)$ show occupancy ratios of the WDW-AD experimental series.

To summarize, the majority of the displacement damage created in both WD-AD and WDW-AD experimental series is occupied with at least one $\mathrm{D}$ at the end of the 2-hour annealing hold for all annealing temperatures except at $800 \mathrm{~K}$. Therefore, the D could potentially affect the annealing 
behaviour of displacement damage at all annealing temperatures used in the experiment except in the $800 \mathrm{~K}$ case. However, as we have observed when comparing the maximum D concentrations measured in our experiment with the ones measured by Markina et al. [4] (see Fig. 4b) no major difference was observed between both experiments. In the case of our experiment, the amount of surviving initial displacement damage when $\mathrm{D}$ was present is only slightly higher compared to the experiment by Markina et al. [4] (Fig. 4b) and was considered to be inside the experimental uncertainties. With this we can conclude that even if D has an effect on defect evolution at elevated temperatures the effect is most likely small or the effect has time-scales which are much shorter than the time-scales used in our experiment.

Other experiments that have studied displacement damage annealing are also available, but unfortunately they cannot be compared to our data for various reasons. For instance, the experiment by Ogorodnikova et al. [50] used two different sample types. One set of samples was re-crystallized and irradiated with $20 \mathrm{MeV}$ W ions before being exposed to the same low-temperature D plasma as we have used, with an exposure temperature of $470 \mathrm{~K}$. This makes it an interesting candidate for comparison, but unfortunately only two data points are available at 700 and $1028 \mathrm{~K}$. The fact that too few data points are available could lead us to a false conclusion when comparing their results to our experiment. The other used samples set was taken from a different manufacturing batch and was used as received without recrystallization. The fact that the samples were not recrystallized makes them difficult to compare to our experiment as the role of lattice defects present from the manufacturing process is indistinguishable from defect created by the irradiation experiment.

Another interesting comparison candidate is the experiment by Simmonds et al. [6]. They have used stress-relieved $\mathrm{W}$ samples, irradiated with $3.4 \mathrm{MeV} \mathrm{Cu}$ ions at various irradiation temperatures and exposed to $110 \mathrm{eV} \mathrm{D}$ plasma at $383 \mathrm{~K}$. Although a comparable D plasma exposure temperature makes it a prime candidate for comparison with our results, their use of elevated temperatures during irradiations instead of post-irradiation annealing makes the comparison impossible. This is because if the irradiation occurs at elevated temperatures a significant contribution to the defect evolution comes from the annihilation between pairs of single vacancies and self-interstitial W atoms (Frenkel-pair annihilation) during irradiation. In the case of defect evolution with post-irradiation annealing (our experiment) Frenkel-pair annihilation is negligible as all of the self-interstitials have already been lost to sinks such as grain-boundaries and the sample surface during the irradiation at room temperature. This makes single-vacancy mobility 
and vacancy cluster dissociation the main contributor to defect evolution during the annealing process (see section 6.1 and 6.2).

The last experiment we will mention here is the experiment by Založnik et al. [5]. The exact same sample preparation and $\mathrm{W}$ irradiation procedure was used as in this work, the only difference being the $1 \mathrm{~h}$ annealing procedure at temperatures ranging from 600 to $1200 \mathrm{~K}$. This makes the experiment a very interesting choice for comparison. Unfortunately, a $0.28 \mathrm{eV} \mathrm{D}$ atom exposure was used to decorate the defects that have survived the annealing process, which forced the use of a relatively high exposure temperature of $500 \mathrm{~K}$. As we have stated in section 6.1 the start of vacancy mobility has been observed in the 500 to $600 \mathrm{~K}$ range [33]-[35], which means that during their $144 \mathrm{~h} \mathrm{D}$ atom exposure, significant single vacancy clustering is expected, which makes a comparison with our results impossible.

Lacking a comparable experiment from the literature we can only state that we expect an effect of $\mathrm{D}$ on displacement damage to be minimal if it exists at all. The effect is expected to be small because the $\mathrm{D}$ de-trapping energies of the various defect type fill-levels and the characteristic energies associated with their annealing evolution coincide perfectly. This means that as the defects start to become mobile or start to dissociate they also start to de-trap their $\mathrm{D}$, meaning that the vast majority of defects are empty during their evolution. As was shown in the discussion of Fig. $3 \mathrm{~b}$ a major part of $\mathrm{D}$ that is lost during annealing is lost in a matter of minutes, while the evolution of defects can occur on much longer time scales as shown in the discussion section of the present work. Therefore, we expect that the D cannot influence the behaviour of the defects in a significant way.

Another important observation of this study is the annealing behaviour of the double damaged samples. Although the trap density is nearly twice as much when samples are damaged while D is present as compared to damaging D-free tungsten, their annealing behaviour is the same. Not only did the total amounts determined by NRA and TDS evolve in the same manner as compared to the samples that were damaged without D being present but the TDS spectra could be described with exactly the same defect types with the same detrapping energies. This in turn bolsters the conclusion from our previous study where it was inferred that the presence of deuterium during displacement damage does not lead to new type of defects but just to an increase in the density of the existing defects. 


\section{Conclusion}

The effect of the presence of $\mathrm{D}$ on the evolution of displacement damage was studied during annealing at elevated temperatures. Displacement damage was introduced by $\mathrm{W}$ irradiation at $295 \mathrm{~K}$ and decorated using a low-temperature D plasma at $370 \mathrm{~K}$. Using a second $\mathrm{W}$ irradiation on half of each sample additional displacement damage was created. After this, samples were successfully annealed at various temperatures ranging from 400 to $800 \mathrm{~K}$ and $\mathrm{D}$ plasma re-exposed at $370 \mathrm{~K}$ to decorate the remaining displacement damage. The samples were analysed using the NRA technique which allowed us to measure the $\mathrm{D}$ depth profile, from which we infer the depth distribution of surviving defects, and by TDS which allowed us to measure how the $\mathrm{D}$ in the sample is distributed within the surviving defects.

The experimental results showed a two-peak desorption structure of the D desorption spectra, where the two peaks behaved independently when annealing at different temperatures. The reduction of $\mathrm{D}$ desorbed in the low-temperature $\mathrm{D}$ desorption peak started in the 500-600 K annealing temperature range while the amount of desorbed $\mathrm{D}$ in the high-temperature peak started between 600 and $800 \mathrm{~K}$. This points to the existence of several defect types that contribute to the overall D retention when samples are exposed to the D plasma at $370 \mathrm{~K}$. As similar desorption peak temperatures, shape and annealing behaviour was observed in both the WD-AD and WDW-AD experimental series we can conclude that the second $\mathrm{W}$ irradiation has not produced any new defect types. Instead only more defects of the existing types have been created. Additionally, based on experimental and simulation results, all defect types anneal in the same manner in both the WD$\mathrm{AD}$ and $\mathrm{WDW}-\mathrm{AD}$ experimental series.

We have performed a simplified simulation with the MHIMS-R code, which is based on the fill-level dependent picture of the hydrogen isotope - defect interaction. The experimental results could be well reproduced by using three different defect types with several fill-levels which were identified as vacancies, small vacancy clusters and large vacancy clusters.

From the results of the simulation it was derived that vacancies start to anneal at around $500 \mathrm{~K}$ and are responsible for the reduction of $\mathrm{D}$ desorption in the low-temperature $\mathrm{D}$ desorption peak observed in the experiment. Small vacancy clusters, which are the cause for D desorption in the high-temperature D desorption peak, started to anneal between 600 and $800 \mathrm{~K}$. Large vacancy clusters, which are responsible for the high-temperature tail in the desorption spectra did not evolve with annealing temperature. 
The effect of $\mathrm{D}$ on displacement damage annealing was difficult to quantify as there is little available information with both $\mathrm{D}$ depth profiles and D desorption spectra in the $300-800 \mathrm{~K}$ annealing temperature range. Still, our results were compared to those of Markina et al. [4] and we found that if there is an effect of $\mathrm{D}$ on displacement damage evolution it is very small for our chosen annealing temperature hold time. The annealing behaviour of samples where displacement damage was created while D was present evolve in the same manner as compared to the samples that were damaged without $\mathrm{D}$ being present. The presence of deuterium during displacement damage does not lead to new type of defects but just of an increase in the density of the existing defects.

\section{Acknowledgement}

This work has been carried out within the framework of the EUROfusion Consortium and has received funding from the Euratom research and training programme 2014-2018 and 2019-2020 under grant agreement No 633053. The views and opinions expressed herein do not necessarily reflect those of the European Commission. The authors acknowledge the support from the Slovenian Research Agency (research core funding No. P2-0405).

\section{References}

[1] J. H. You, "A review on two previous divertor target concepts for DEMO: Mutual impact between structural design requirements and materials performance," Nucl. Fusion, vol. 55, no. 11, p. 113026, 2015.

[2] Y. Hatano et al., "Deuterium trapping at defects created with neutron and ion irradiations in tungsten," Nucl. Fusion, vol. 53, no. 7, p. 073006, 2013.

[3] W. R. Wampler and R. P. Doerner, "The influence of displacement damage on deuterium retention in tungsten exposed to plasma," Nucl. Fusion, vol. 49, no. 11, p. 115023, 2009.

[4] E. Markina, M. Mayer, A. Manhard, and T. Schwarz-Selinger, "Recovery temperatures of defects in tungsten created by self-implantation," J. Nucl. Mater., vol. 463, pp. 329-332, 2015.

[5] A. Založnik et al., "The influence of the annealing temperature on deuterium retention in self-damaged tungsten," in Physica Scripta, 2016, vol. 2016, no. T167, p. 014031.

[6] M. J. Simmonds et al., "Reduced deuterium retention in simultaneously damaged and annealed tungsten," J. Nucl. Mater., vol. 494, pp. 67-71, Oct. 2017. 
[7] T. Schwarz-Selinger, J. Bauer, S. Elgeti, and S. Markelj, "Influence of the presence of deuterium on displacement damage in tungsten," Nucl. Mater. Energy, vol. 17, pp. 228-234, Dec. 2018.

[8] S. Markelj et al., "Deuterium retention in tungsten simultaneously damaged by high energy W ions and loaded by D atoms," Nucl. Mater. Energy, vol. 12, pp. 169-174, Aug. 2017.

[9] S. Markelj et al., "Displacement damage stabilization by hydrogen presence under simultaneous W ion damage and D ion exposure," Nucl. Fusion, vol. 59, no. 8, p. 086050 , Aug. 2019.

[10] E. A. Hodille et al., "Stabilization of defects by the presence of hydrogen in tungsten: simultaneous W-ion damaging and D-atom exposure," Nucl. Fusion, vol. 59, no. 1, p. 016011, Jan. 2018.

[11] M. Pečovnik, S. Markelj, A. Založnik, and T. Schwarz-Selinger, "Influence of grain size on deuterium transport and retention in self-damaged tungsten," J. Nucl. Mater., vol. 513, pp. 198-208, 2019.

[12] D. Kato, H. Iwakiri, Y. Watanabe, K. Morishita, and T. Muroga, "Super-saturated hydrogen effects on radiation damages in tungsten under the high-flux divertor plasma irradiation," Nucl. Fusion, vol. 55, no. 8, p. 083019, Aug. 2015.

[13] E. Hayward and C. Deo, "Energetics of small hydrogen-vacancy clusters in bec iron," $J$. Phys. Condens. Matter, vol. 23, no. 42, p. 425402, Oct. 2011.

[14] J. Ziegler, "SRIM.” [Online]. Available: http://srim.org/.

[15] O. V. Ogorodnikova and V. Gann, "Simulation of neutron-induced damage in tungsten by irradiation with energetic self-ions," J. Nucl. Mater., vol. 460, pp. 60-71, May 2015.

[16] A. Manhard, T. Schwarz-Selinger, and W. Jacob, "Quantification of the deuterium ion fluxes from a plasma source," Plasma Sources Sci. Technol., vol. 20, no. 1, p. 015010, 2011.

[17] S. Kapser et al., "Influence of sub-surface damage evolution on low-energy-plasma-driven deuterium permeation through tungsten," Nucl. Fusion, vol. 58, no. 5, p. 056027, May 2018.

[18] M. Pečovnik, E. A. Hodille, T. Schwarz-Selinger, C. Grisolia, and S. Markelj, "New rate equation model to describe the stabilization of displacement damage by hydrogen atoms during ion irradiation in tungsten," Nucl. Fusion, vol. 60, p. 036024, 2020.

[19] S. Markelj, A. Založnik, and I. Čadež, "Interaction of ammonia and hydrogen with tungsten at elevated temperature studied by gas flow through a capillary," J. Vac. Sci. Technol. A 
Vacuum, Surfaces, Film., vol. 35, no. 6, p. 061602, Nov. 2017.

[20] K. Schmid and U. Von Toussaint, "Statistically sound evaluation of trace element depth profiles by ion beam analysis," Nucl. Instruments Methods Phys. Res. Sect. B Beam Interact. with Mater. Atoms, vol. 281, pp. 64-71, 2012.

[21] L. K. Keys and J. Moteff, "Neutron irradiation and defect recovery of tungsten," J. Nucl. Mater., vol. 34, pp. 260-280, 1970.

[22] B. Wielunska, M. Mayer, T. Schwarz-Selinger, U. Von Toussaint, and J. Bauer, "Cross section data for the $\mathrm{D}(3 \mathrm{He}, \mathrm{p}) 4 \mathrm{He}$ nuclear reaction from 0.25 to $6 \mathrm{MeV}$," Nucl. Instruments Methods Phys. Res. Sect. B Beam Interact. with Mater. Atoms, vol. 371, pp. 41-45, 2016.

[23] E. A. Hodille et al., "Study of hydrogen isotopes behavior in tungsten by a multi trapping macroscopic rate equation model," in Physica Scripta, 2016, vol. 2016, no. T167, p. 014011.

[24] K. Schmid, U. Von Toussaint, and T. Schwarz-Selinger, "Transport of hydrogen in metals with occupancy dependent trap energies," J. Appl. Phys., vol. 116, no. 13, p. 134901, Oct. 2014.

[25] K. Schmid et al., "Recent progress in the understanding of $\mathrm{H}$ transport and trapping in W," in Physica Scripta, 2017, vol. 2017, no. T170, p. 014037.

[26] O. V. Ogorodnikova, J. Roth, and M. Mayer, "Ion-driven deuterium retention in tungsten," J. Appl. Phys., vol. 103, no. 3, p. 034902, Feb. 2008.

[27] N. Fernandez, Y. Ferro, and D. Kato, "Hydrogen diffusion and vacancies formation in tungsten: Density Functional Theory calculations and statistical models," Acta Mater., vol. 94, pp. 307-318, Aug. 2015.

[28] E. A. Hodille et al., "Simulations of atomic deuterium exposure in self-damaged tungsten," Nucl. Fusion, vol. 57, no. 5, p. 056002, May 2017.

[29] A. Založnik, S. Markelj, T. Schwarz-Selinger, and K. Schmid, "Deuterium atom loading of self-damaged tungsten at different sample temperatures," J. Nucl. Mater., vol. 496, pp. 1-8, 2017.

[30] K. Heinola, T. Ahlgren, K. Nordlund, and J. Keinonen, "Hydrogen interaction with point defects in tungsten," Phys. Rev. B - Condens. Matter Mater. Phys., vol. 82, no. 9, p. 094102, Sep. 2010.

[31] D. F. Johnson and E. A. Carter, "Hydrogen in tungsten: Absorption, diffusion, vacancy trapping, and decohesion," J. Mater. Res., vol. 25, no. 2, pp. 315-327, Feb. 2010. 
[32] J. Hou, X. S. Kong, X. Wu, J. Song, and C. S. Liu, "Predictive model of hydrogen trapping and bubbling in nanovoids in bcc metals," Nat. Mater., vol. 18, no. 8, pp. 833-839, Aug. 2019.

[33] H. Eleveld and A. van Veen, "Void growth and thermal desorption of deuterium from voids in tungsten," J. Nucl. Mater., vol. 212-215, P, pp. 1421-1425, 1994.

[34] A. Debelle, M. F. Barthe, and T. Sauvage, "First temperature stage evolution of irradiationinduced defects in tungsten studied by positron annihilation spectroscopy," J. Nucl. Mater., vol. 376, no. 2, pp. 216-221, 2008.

[35] J. de Vries, "Positron lifetime technique with applications in materials science," Technische Universiteit Delft, The Netherlands, 1987.

[36] M. Zibrov, "The Influence of Radiation, Mechanical, and Plasma-Induced Damage on Deuterium Retention in Tungsten Mikhail Zibrov,” Technische Universität München, Universiteit Gent, 2018.

[37] S. K.-D., Rasch; R. W., Siegel; H., "Quenching and recovery investigations of vacancies in tungsten," Philos. Mag. A, vol. 41, no. 1, pp. 91-117, 1980.

[38] G. Y. Huang, N. Juslin, and B. D. Wirth, "First-principles study of vacancy, interstitial, noble gas atom interstitial and vacancy clusters in bcc-W," Comput. Mater. Sci., vol. 123, pp. 121-130, 2016.

[39] K. Heinola, F. Djurabekova, and T. Ahlgren, "On the stability and mobility of di-vacancies in tungsten," Nucl. Fusion, vol. 58, no. 2, p. 026004, Feb. 2018.

[40] M. Muzyk, D. Nguyen-Manh, K. J. Kurzydłowski, N. L. Baluc, and S. L. Dudarev, "Phase stability, point defects, and elastic properties of W-V and W-Ta alloys," Phys. Rev. B Condens. Matter Mater. Phys., vol. 84, no. 10, p. 104115, Sep. 2011.

[41] D. R. Mason, X. Yi, M. A. Kirk, and S. L. Dudarev, "Elastic trapping of dislocation loops in cascades in ion-irradiated tungsten foils," J. Phys. Condens. Matter, vol. 26, no. 37, p. 375701, 2014.

[42] D. R. Mason, D. Nguyen-Manh, and C. S. Becquart, "An empirical potential for simulating vacancy clusters in tungsten," J. Phys. Condens. Matter, vol. 29, no. 50, p. 505501, Dec. 2017.

[43] C. S. Becquart, C. Domain, U. Sarkar, A. Debacker, and M. Hou, "Microstructural evolution of irradiated tungsten: Ab initio parameterisation of an OKMC model," J. Nucl. Mater., vol. 
403, no. 1-3, pp. 75-88, 2010.

[44] A. De Backer et al., "Multiscale modelling of the interaction of hydrogen with interstitial defects and dislocations in BCC tungsten," Nucl. Fusion, vol. 58, no. 1, p. 016006, Jan. 2018.

[45] Y. N. Osetsky, D. J. Bacon, A. Serra, B. N. Singh, and S. I. Golubov, "Stability and mobility of defect clusters and dislocation loops in metals," J. Nucl. Mater., vol. 276, no. 1, pp. 6577, 2000.

[46] B. D. Wirth, G. R. Odette, D. Maroudas, and G. E. Lucas, "Dislocation loop structure, energy and mobility of self-interstitial atom clusters in bcc iron," J. Nucl. Mater., vol. 276, no. 1, pp. 33-40, 2000.

[47] B. D. Wirth, G. R. Odette, D. Maroudas, and G. E. Lucas, "Energetics of formation and migration of self-interstitials and self-interstitial clusters in $\alpha$-iron," J. Nucl. Mater., vol. 244, no. 3, pp. 185-194, 1997.

[48] A. V. Barashev, S. I. Golubov, and H. Trinkaus, "Reaction kinetics of glissile interstitial clusters in a crystal containing voids and dislocations," Philos. Mag. A Phys. Condens. Matter, Struct. Defects Mech. Prop., vol. 81, no. 10, pp. 2515-2532, Oct. 2001.

[49] W. Chrominski, L. Ciupinski, P. Bazarnik, S. Markelj, and T. Schwarz-Selinger, "TEM investigation of the influence of dose rate on radiation damage and deuterium retention in tungsten," Mater. Charact., vol. 154, pp. 1-6, 2019.

[50] O. V. Ogorodnikova, Y. Gasparyan, V. Efimov, Ciupiński, and J. Grzonka, "Annealing of radiation-induced damage in tungsten under and after irradiation with $20 \mathrm{MeV}$ self-ions," $J$. Nucl. Mater., vol. 451, no. 1-3, pp. 379-386, Aug. 2014. 